\title{
STRESS FIELD NEAR THE TIP OF A CRACK IN A POROELASTIC TRANSVERSELY ANISOTROPIC SATURATED ROCK
}

\author{
Antonio Bobet \\ School of Civil Engineering, Purdue University, West Lafayette, IN, USA \\ Haitao $\mathrm{Yu}^{*}$ \\ Key Laboratory of Geotechnical and Underground Engineering of \\ Ministry of Education, Tongji University, Shanghai, China \& \\ Department of Civil Engineering, Shanghai Jiaotong University, Shanghai, China
}

\begin{abstract}
Closed-form solutions are obtained for the singular stress and displacement fields at the tip of a single crack in an infinite poroelastic transversely anisotropic saturated medium. Three different scenarios are considered: dry medium (no pore pressures), undrained loading (no dissipation of excess pore pressures), and drained loading (all excess pore pressures have dissipated). The results show that the stresses around the tip of a pressurized crack in a saturated medium with drained loading are smaller than those produced when the medium is dry. Undrained loading reduces the effective stresses at the tip while it increases the excess pore pressures.
\end{abstract}

Keywords: poroelasticy, anisotropy, LEFM, undrained loading, drained loading

\section{Notation}

The following is a list of the variables used in the paper:

a

b

$E_{\mathrm{x}}, \mathrm{E}_{\mathrm{y}}$

$\mathrm{G}_{\mathrm{xy}}$

$\mathrm{K}_{\mathrm{I}}, \mathrm{K}_{\mathrm{II}}$

$\mathrm{k}_{\mathrm{x}}, \mathrm{k}_{\mathrm{y}}$

$\mathrm{k}_{\xi \xi}, \mathrm{k}_{\xi \eta} \mathrm{k}_{\xi \eta}$

$\mathrm{M}$

$\mathrm{Q}$ half the length of the crack or half the length of the major axis of an ellipse half the length of the minor axis of an ellipse

Young's modulus of the medium in $x-y$ coordinates

shear modulus of the medium in $\mathrm{x}-\mathrm{y}$ coordinates

Mode I and II Stress Intensity Factors

permeabilities of the medium in $\mathrm{x}-\mathrm{y}$ coordinates

permeabilities of the medium in $\xi-\eta$ coordinates

Biot's modulus

flow

*Corresponding author. Tel.: +86 21 65982005; fax: +86 2165984573.

E-mail address: yuhaitao@tongji.edu.cn (Haitao Yu). 


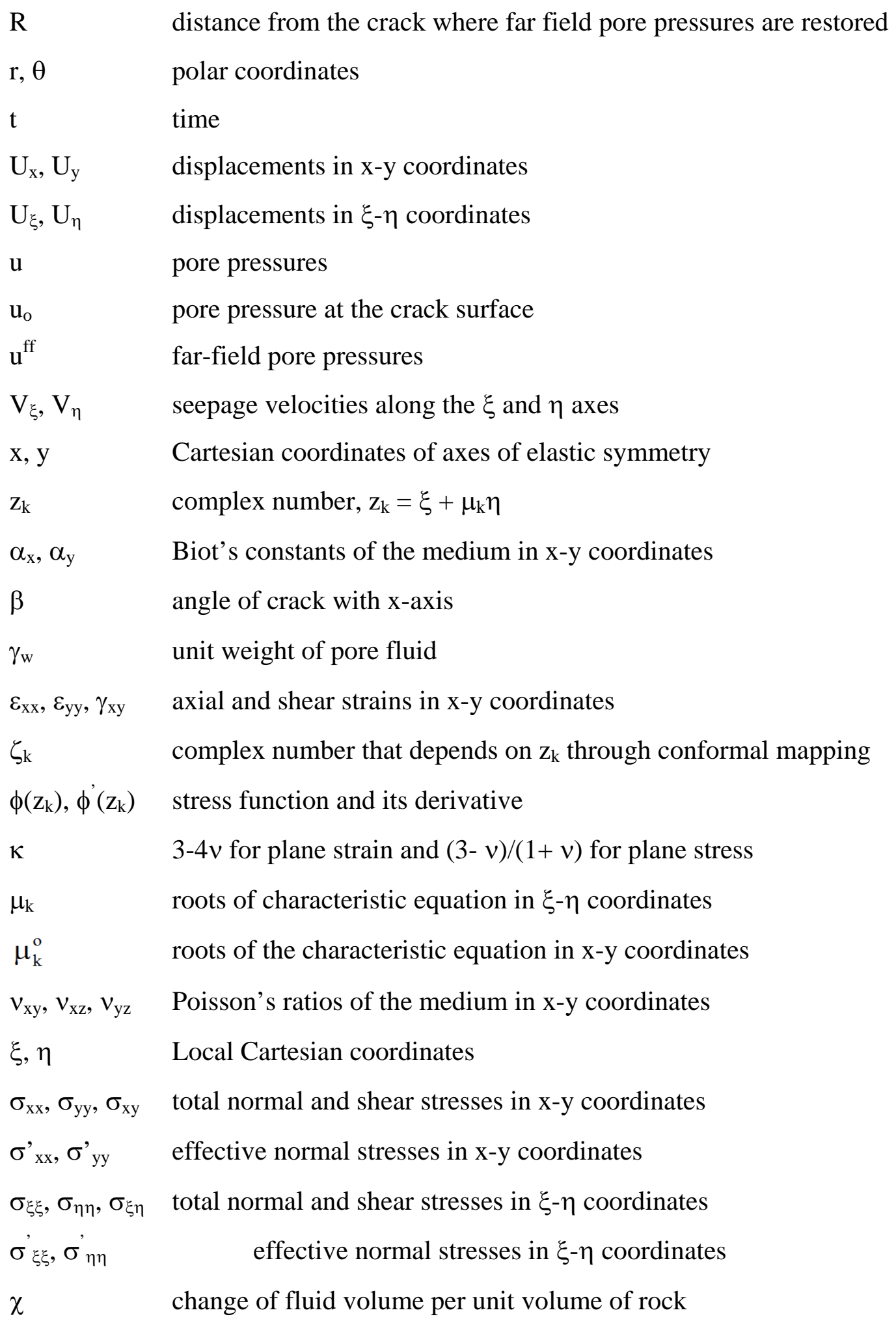




\section{Introduction}

The stability of rock masses is determined by the presence of joints or discontinuities. These joints, under certain loading conditions, can open or close, slide, propagate as new joints or cracks, and coalesce. Discontinuities, in brittle stiff rock materials, can be treated as cracks within the framework of LEFM or Linear Elastic Fracture Mechanics (Whittaker et al., 1992; Mutlu and Bobet, 2006; Kao et al., 2008; Hedayat et al., 2014). The presence of a crack affects significantly the state of stress in a medium and in particular at its tip (e.g. Griffith, 1920; McClintock and Walsh, 1962; Liebowitz, 1968; Wang, 2000). It has been shown (e.g. Whittaker et al., 1992; Bobet, 2000) that the stress field near the tip of a crack can be correlated with initiation of tensile and shear cracks. Common initiation theories such as the maximum tangential stress theory (Erdogan and Sih, 1963), the maximum energy release rate theory (Griffith, 1921), the minimum energy density theory (Sih, 1974), as well as others (e.g. Shen and Stephansson, 1994; Bobet, 2000) require or are based on detailed knowledge of the stresses near the tip as well as the values of the Stress Intensity Factors (SIFs). Most closed-form solutions for the SIFs have been obtained for an isolated crack in an infinite, homogeneous, isotropic and elastic medium. While it may be acceptable to assume LEFM for hard rocks and to some extent homogeneity, at least at the scale of the crack, rocks are often anisotropic. Efforts have been made to obtain analytical solutions for a crack in an anisotropic elastic body. Sih et al. (1965) used complex variable theory to provide the SIFs and near-tip stress and displacement fields for a crack in an anisotropic elastic medium for loading modes I, II and III. The expressions were used by Chen et al. (1998) to verify a new Boundary Element method code that was then used to investigate fracturing of anisotropic rocks by diametral compression. Additional theoretical solutions have been utilized by other authors to calculate SIFs at sharp notches in anisotropic media (Wu and Chang, 1993; Labossiere and Dunn, 1998; Ju et al., 2010). However analytical solutions are often limited to those cases that are treatable and usually a numerical solution may be needed (e.g. Chen et al., 1998; Su and Sun, 2003).

Additional complexity arises because most fracture phenomena, from tunneling to hydraulic fracturing, occur in saturated rock masses below the water table. The response 
of the rock depends on how fast the dissipation of excess pore pressures, which may be induced by loading, occurs. Two limiting scenarios are usually contemplated in geomechanics: drained and undrained loading. In the first scenario, the loading is applied at a rate small enough such that dissipation of excess pore pressures occurs very quickly and as the load is applied. This could be the case for rock materials with very high matrix permeability. The second scenario takes place when the loading rate is fast enough such that no excess pore pressures are dissipated, and may occur in rock materials with very small matrix permeability. This paper builds on the theoretical framework that already exists for cracks in isotropic and anisotropic media and provides new closed-form solutions for the SIFs and/or for the full first-term expressions for stresses and displacements of a crack in a two-dimensional plane strain, infinite, transversely anisotropic poroelastic medium subjected to mixed mode I-II loading, and for the following conditions: saturated medium under undrained loading and saturated medium under drained loading. It also provides numerical results and a discussion for the case of a pressurized crack (i.e. hydraulic fracturing). In the following, the mechanics sign convention is used with tension positive and compression negative.

\section{General Formulation}

Equations (1) provide the first-term approximation for two dimensional stress and displacement fields around the tip of a crack in an isotropic and homogeneous medium for loading modes I and II.

$$
\begin{aligned}
& \left\{\begin{array}{l}
\sigma_{\xi \xi} \\
\sigma_{\eta \eta} \\
\sigma_{\xi \eta}
\end{array}\right\}=\frac{\mathrm{K}_{\mathrm{I}}}{\sqrt{2 \pi \mathrm{r}}} \cos \frac{\theta}{2}\left\{\begin{array}{c}
1-\sin \frac{\theta}{2} \sin \frac{3 \theta}{2} \\
1+\sin \frac{\theta}{2} \sin \frac{3 \theta}{2} \\
\sin \frac{\theta}{2} \cos \frac{3 \theta}{2}
\end{array}\right\}+\frac{\mathrm{K}_{\mathrm{II}}}{\sqrt{2 \pi \mathrm{r}}}\left\{\begin{array}{c}
-\sin \frac{\theta}{2}\left(2+\cos \frac{\theta}{2} \cos \frac{3 \theta}{2}\right) \\
\sin \frac{\theta}{2} \cos \frac{\theta}{2} \cos \frac{3 \theta}{2} \\
\cos \frac{\theta}{2}\left(1-\sin \frac{\theta}{2} \sin \frac{3 \theta}{2}\right)
\end{array}\right\} \\
& \left\{\begin{array}{l}
\mathrm{U}_{\xi} \\
\mathrm{U}_{\eta}
\end{array}\right\}=\frac{\mathrm{K}_{\mathrm{I}}}{4 \mathrm{G}} \sqrt{\frac{\mathrm{r}}{2 \pi}}\left\{\begin{array}{l}
(2 \kappa-1) \cos \frac{\theta}{2}-\cos \frac{3 \theta}{2} \\
(2 \kappa+1) \sin \frac{\theta}{2}-\sin \frac{3 \theta}{2}
\end{array}\right\}+\frac{\mathrm{K}_{\mathrm{II}}}{4 \mathrm{G}} \sqrt{\frac{\mathrm{r}}{2 \pi}}\left\{\begin{array}{l}
(2 \kappa+3) \sin \frac{\theta}{2}+\sin \frac{3 \theta}{2} \\
-(2 \kappa-3) \cos \frac{\theta}{2}-\cos \frac{3 \theta}{2}
\end{array}\right\}
\end{aligned}
$$


where $\sigma_{\xi \xi}, \sigma_{\eta \eta}, \sigma_{\xi \eta}$ are stresses and $U_{\xi}$ and $U_{\eta}$ displacements in the Cartesian coordinate system shown in Figure 1; $\mathrm{r}$ and $\theta$ are the Cartesian coordinates, $\mathrm{K}_{\mathrm{I}}$ and $\mathrm{K}_{\mathrm{II}}$ are the stress intensity factors; $\kappa=3-4 v$ for plane strain and $(3-v) /(1+v)$ for plane stress and $G$ is the shear modulus. Clearly the solutions apply only to dry material since, for their derivation, no assumptions regarding water or water flow were made.

Figure 2 shows a two-dimensional elliptical crack in an infinite, transversely anisotropic medium. The axes of anisotropic symmetry are $x-y$, with the coordinate system associated with the crack $\xi, \eta$, which makes an angle $\beta$ with the $x-y$ axes. In the $x-y$ axis, strains and stresses are, in plane strain, given by (e.g. Detournay and Cheng, 1993; Ting, 1996; Cheng, 1998; Wang 2000; Bobet, 2011):

$$
\begin{aligned}
& \varepsilon_{\mathrm{xx}}=\alpha_{1} \sigma_{\mathrm{xx}}-\alpha_{2} \sigma_{\mathrm{yy}}+\beta_{1} \mathrm{u} \\
& \varepsilon_{\mathrm{yy}}=-\alpha_{2} \sigma_{\mathrm{xx}}+\alpha_{3} \sigma_{\mathrm{yy}}+\beta_{2} \mathrm{u} \\
& \gamma_{\mathrm{xy}}=\frac{\tau_{\mathrm{xy}}}{\mathrm{G}_{\mathrm{xy}}} \\
& \alpha_{1}=\frac{1-v_{\mathrm{xz}}^{2}}{\mathrm{E}_{\mathrm{x}}} \\
& \alpha_{2}=\frac{\left(1+v_{\mathrm{xz}}\right) v_{\mathrm{yx}}}{\mathrm{E}_{\mathrm{y}}} \\
& \alpha_{3}=\left(1-\frac{\mathrm{E}_{\mathrm{x}}}{\mathrm{E}_{\mathrm{y}}} v_{\mathrm{yx}}^{2}\right) \frac{1}{\mathrm{E}_{\mathrm{y}}} \\
& \beta_{1}=\alpha_{1} \alpha_{\mathrm{x}}-\alpha_{2} \alpha_{\mathrm{y}} \\
& \beta_{2}=-\alpha_{2} \alpha_{\mathrm{x}}+\alpha_{3} \alpha_{\mathrm{y}}
\end{aligned}
$$

where $\sigma_{x x}, \sigma_{y y}, \tau_{x y}$ are the total stresses along the x- and y-axis (Figure 2) and the shear stresses, respectively; $\varepsilon_{\mathrm{xx}}, \varepsilon_{\mathrm{yy}}, \gamma_{\mathrm{xy}}$ are the strains; $\mathrm{E}_{\mathrm{x}}$ and $\mathrm{E}_{\mathrm{y}}$ are the Young's modulus in the $\mathrm{x}$ and $\mathrm{y}$ directions, $v_{\mathrm{xz}}$ and $v_{\mathrm{yx}}$ are the Poisson's ratios in the $\mathrm{xz}$ and $\mathrm{yx}$ directions, respectively, and $G_{x y}$ is the shear modulus. Note that, because of the symmetry of the strain tensor, $v_{\mathrm{xy}}=v_{\mathrm{yx}} \mathrm{E}_{\mathrm{x}} / \mathrm{E}_{\mathrm{y}}$. Note also that the properties in the $\mathrm{z}$ and $\mathrm{x}$ directions are the same. $\alpha_{\mathrm{x}}$ and $\alpha_{\mathrm{y}}$ are the Biot's constants in the $\mathrm{x}$ and $\mathrm{y}$ directions, and $\mathrm{u}$ is the pore pressure. 
Equilibrium is written in terms of total stresses and is expressed as:

$$
\begin{aligned}
& \frac{\partial \sigma_{x x}}{\partial x}+\frac{\partial \tau_{x y}}{\partial y}=0 \\
& \frac{\partial \sigma_{y y}}{\partial y}+\frac{\partial \tau_{x y}}{\partial x}=0
\end{aligned}
$$

and pore pressures must obey the following field equation:

$$
\mathrm{k}_{\mathrm{x}} \frac{\partial^{2} \mathrm{u}}{\partial \mathrm{x}^{2}}+\mathrm{k}_{\mathrm{y}} \frac{\partial^{2} \mathrm{u}}{\partial \mathrm{y}^{2}}=\gamma_{\mathrm{w}} \frac{\partial \chi}{\partial \mathrm{t}}
$$

where $\mathrm{k}_{\mathrm{x}}$ and $\mathrm{k}_{\mathrm{y}}$ are the permeabilities in the $\mathrm{x}$ and $\mathrm{y}$ axes, respectively, $\gamma_{\mathrm{w}}$ is the unit weight of the pore fluid, and $\chi$ is the change of fluid volume per unit volume of the porous material. In addition,

$\mathrm{u}=\mathrm{M}\left(\chi-\alpha_{\mathrm{x}} \varepsilon_{\mathrm{xx}}-\alpha_{\mathrm{y}} \varepsilon_{\mathrm{yy}}\right)$

where $\mathrm{M}$ is the Biot's modulus, defined as the increase of the amount of fluid per unit volume of rock as a result of a unit increase of pore pressure under constant volumetric strain.

It is convenient to express equilibrium and stress-strain relations in the coordinate system, $\xi-\eta$. The equations take the form:

$$
\begin{aligned}
& \frac{\partial \sigma_{\xi \xi}}{\partial \xi}+\frac{\partial \tau_{\xi \eta}}{\partial \eta}=0 \\
& \frac{\partial \sigma_{\eta \eta}}{\partial \eta}+\frac{\partial \tau_{\xi \eta}}{\partial \xi}=0
\end{aligned}
$$

where $\sigma_{\xi \xi}, \sigma_{\eta \eta}, \tau_{\xi \eta}$ are the total stresses and shear stresses along the $\xi$ - and $\eta$-axis. Strains are: 


$$
\begin{aligned}
& \varepsilon_{\xi \xi}=a_{11} \sigma_{\xi \xi}+a_{12} \sigma_{\eta \eta}+a_{13} \sigma_{\xi \eta}+\left(\beta_{1} \cos ^{2} \beta+\beta_{2} \sin ^{2} \beta\right) u \\
& \varepsilon_{\eta \eta}=a_{21} \sigma_{\xi \xi}+a_{22} \sigma_{\eta \eta}+a_{23} \sigma_{\xi \eta}+\left(\beta_{1} \sin ^{2} \beta+\beta_{2} \cos ^{2} \beta\right) u \\
& \gamma_{\xi \eta}=a_{31} \sigma_{\xi \xi}+a_{32} \sigma_{\eta \eta}+a_{33} \sigma_{\xi \eta}+\left(\beta_{2}-\beta_{1}\right) \sin 2 \beta u \\
& a_{11}=\alpha_{1} \cos ^{4} \beta+\alpha_{3} \sin ^{4} \beta+\left(\frac{1}{G_{x y}}-2 \alpha_{2}\right) \sin ^{2} \beta \cos ^{2} \beta \\
& a_{12}=a_{21}=-\left[\alpha_{2}\left(\cos ^{4} \beta+\sin ^{4} \beta\right)+\left(\frac{1}{G_{x y}}-\alpha_{1}-\alpha_{3}\right) \sin ^{2} \beta \cos ^{2} \beta\right] \\
& a_{13}=a_{31}=\left[\left(\frac{1}{G_{x y}}-2 \alpha_{1}\right) \cos ^{2} \beta-\left(\frac{1}{G_{x y}}-2 \alpha_{3}\right) \sin ^{2} \beta-2 \alpha_{2} \cos 2 \beta\right] \sin \beta \cos \beta \\
& a_{22}=\alpha_{3} \cos ^{4} \beta+\alpha_{1} \sin ^{4} \beta+\left(\frac{1}{G_{x y}}-2 \alpha_{2}\right) \sin ^{2} \beta \cos ^{2} \beta \\
& a_{23}=a_{32}=\left[\left(\frac{1}{G_{x y}}-2 \alpha_{1}\right) \sin ^{2} \beta-\left(\frac{1}{G_{x y}}-2 \alpha_{3}\right) \cos ^{2} \beta+2 \alpha_{2} \cos 2 \beta\right] \sin \beta \cos \beta \\
& a_{33}=\left(\alpha_{1}+2 \alpha_{2}+\alpha_{3}\right) \sin ^{2} 2 \beta+\frac{1}{G_{x y}} \cos ^{2} 2 \beta
\end{aligned}
$$

Equilibrium is satisfied if a stress function $\mathrm{F}(\mathrm{x}, \mathrm{y})$ is found such that (Lekhnitskii, 1963):

$$
\begin{aligned}
\sigma_{\xi \xi} & =\frac{\partial^{2} \mathrm{~F}}{\partial \eta^{2}} \\
\sigma_{\eta \eta} & =\frac{\partial^{2} \mathrm{~F}}{\partial \xi^{2}} \\
\tau_{\xi \eta} & =-\frac{\partial^{2} \mathrm{~F}}{\partial \xi \partial \eta}
\end{aligned}
$$

The compatibility equation can be written, in terms of the function $\mathrm{F}(\mathrm{x}, \mathrm{y})$ as:

$$
\begin{aligned}
& \mathrm{a}_{11} \frac{\partial^{4} \mathrm{~F}}{\partial \eta^{4}}+\mathrm{a}_{22} \frac{\partial^{4} \mathrm{~F}}{\partial \xi^{4}}-2 \mathrm{a}_{13} \frac{\partial^{4} \mathrm{~F}}{\partial \xi \partial \eta^{3}}+\left(2 \mathrm{a}_{12}+\mathrm{a}_{33}\right) \frac{\partial^{4} \mathrm{~F}}{\partial \xi^{2} \partial \eta^{2}}-2 \mathrm{a}_{23} \frac{\partial^{4} \mathrm{~F}}{\partial \xi^{3} \partial \eta}= \\
& -\left(\beta_{1} \sin ^{2} \beta+\beta_{2} \cos ^{2} \beta\right) \frac{\partial^{2} \mathrm{u}}{\partial \xi^{2}}-\left(\beta_{1} \cos ^{2} \beta+\beta_{2} \sin ^{2} \beta\right) \frac{\partial^{2} \mathrm{u}}{\partial \eta^{2}}+\left(\beta_{2}-\beta_{1}\right) \sin 2 \beta \frac{\partial^{2} \mathrm{u}}{\partial \xi \partial \eta}
\end{aligned}
$$

The solution of equation (9) provides $\mathrm{F}(\mathrm{x}, \mathrm{y})$, and hence stresses and displacements can be obtained from (8) and integration of (7). Lekhnitskii (1963) proposed a solution of (9) by 
introducing the complex variable $\mathrm{z}_{\mathrm{k}}=\xi+\mu_{\mathrm{k}} \eta$, where $\mu_{\mathrm{k}}$ is a complex number. Expressing (9) as a function of the complex variable $z_{k}$, one gets:

$$
\begin{aligned}
& {\left[a_{11} \mu_{k}^{4}-2 a_{13} \mu_{k}^{3}+\left(a_{33}+2 a_{12}\right) \mu_{k}^{2}-2 a_{23} \mu_{k}+a_{22}\right] \frac{\partial^{4} \mathrm{~F}}{\partial z^{4}}=} \\
& -\left(\beta_{1} \sin ^{2} \beta+\beta_{2} \cos ^{2} \beta\right) \frac{\partial^{2} u}{\partial \xi^{2}}-\left(\beta_{1} \cos ^{2} \beta+\beta_{2} \sin ^{2} \beta\right) \frac{\partial^{2} u}{\partial \eta^{2}}+\left(\beta_{2}-\beta_{1}\right) \sin 2 \beta \frac{\partial^{2} u}{\partial \xi \partial \eta}
\end{aligned}
$$

By introducing the function $\phi\left(\mathrm{z}_{\mathrm{k}}\right)=\mathrm{F}^{\prime}\left(\mathrm{z}_{\mathrm{k}}\right)=\mathrm{dF} / \mathrm{d \textrm {z } _ { \mathrm { k } }}$, the total stresses can be obtained from:

$$
\begin{aligned}
& \sigma_{\xi \xi}=2 \operatorname{Re}\left[\mu_{1}^{2} \phi_{1}^{\prime}\left(z_{1}\right)+\mu_{2}^{2} \phi_{2}^{\prime}\left(z_{2}\right)\right]+\frac{\partial^{2} F_{o}}{\partial \eta^{2}} \\
& \sigma_{\eta \eta}=2 \operatorname{Re}\left[\phi_{1}^{\prime}\left(z_{1}\right)+\phi_{2}^{\prime}\left(z_{2}\right)\right]+\frac{\partial^{2} F_{o}}{\partial \xi^{2}} \\
& \tau_{\xi \eta}=-2 \operatorname{Re}\left[\mu_{1} \phi_{1}^{\prime}\left(z_{1}\right)+\mu_{2} \phi_{2}^{\prime}\left(z_{2}\right)\right]-\frac{\partial^{2} F_{o}}{\partial \xi \partial \eta}
\end{aligned}
$$

where $F_{0}$ is a particular solution of (9) and $\mu_{1}$ and $\mu_{2}$ are the roots of the characteristic equation:

$$
\left[\mathrm{a}_{11} \mu_{\mathrm{k}}^{4}-2 \mathrm{a}_{13} \mu_{\mathrm{k}}^{3}+\left(\mathrm{a}_{33}+2 \mathrm{a}_{12}\right) \mu_{\mathrm{k}}^{2}-2 \mathrm{a}_{23} \mu_{\mathrm{k}}+\mathrm{a}_{22}\right]=0
$$

Lekhnitskii (1963) showed that the roots of (12) are always complex numbers and can be expressed as:

$$
\begin{array}{ll}
\mu_{\mathrm{k}}=\mu_{\mathrm{k} 1}+\mathrm{i} \mu_{\mathrm{k} 2}=\frac{\mu_{\mathrm{k}}^{\mathrm{o}} \cos \beta-\sin \beta}{\cos \beta+\mu_{\mathrm{k}}^{\mathrm{o}} \sin \beta}, \quad \mathrm{k}=1,2 \\
\bar{\mu}_{\mathrm{k}}=\mu_{\mathrm{k} 1}-\mathrm{i} \mu_{\mathrm{k} 2}=\frac{\bar{\mu}_{\mathrm{k}}^{\mathrm{o}} \cos \beta-\sin \beta}{\cos \beta+\bar{\mu}_{\mathrm{k}}^{\mathrm{o}} \sin \beta}, \quad \mathrm{k}=1,2
\end{array}
$$

where $\mu_{\mathrm{k}}^{\mathrm{o}}$, and its conjugate $\bar{\mu}_{\mathrm{k}}^{\mathrm{o}}$, are the roots of the characteristic equation in the $\mathrm{x}-\mathrm{y}$ coordinate system, i.e. the roots of the expression: 
$\alpha_{1} \mu_{\mathrm{k}}^{4}+\left(\frac{1}{\mathrm{G}_{\mathrm{xy}}}-2 \alpha_{2}\right) \mu_{\mathrm{k}}^{2}+\alpha_{3}=0$

Effective stresses, total stresses and pore pressures in each coordinate system can be obtained as follows:

$$
\begin{aligned}
& \sigma_{x x}^{\prime}=\sigma_{x x}+\alpha_{x} u \\
& \sigma_{y y}^{\prime}=\sigma_{y y}+\alpha_{y} u \\
& \sigma_{\xi \xi}^{\prime}=\sigma_{\xi \xi}+\left(\alpha_{x} \cos ^{2} \beta+\alpha_{y} \sin ^{2} \beta\right) u \\
& \sigma_{\eta \eta}^{\prime}=\sigma_{\eta \eta}+\left(\alpha_{x} \sin ^{2} \beta+\alpha_{y} \cos ^{2} \beta\right) u
\end{aligned}
$$

\section{Crack in dry transversely anisotropic elastic rock}

In this case, the right-hand side of Equation (9) is zero. The problem that is solved is that of an elliptical crack with dimensions 2a long and 2b wide, as shown in Figures 2 and 3. The solution for a crack follows by making $b=0$. This approach is different than that taken by Sih et al. (1965), and has the advantage that is general enough such that solutions involving fluid pressure can be reached, as it will be discussed in the next sections. The solution is included here for completeness and as an example of the general approach taken in the paper. Evidently both methods give the same correct solution.

The stress functions $\phi_{1}$ and $\phi_{2}$, for an elliptical opening, following Lekhnitskii's (1963), are given by:

$$
\begin{aligned}
& \phi_{1}\left(z_{1}\right)=\frac{1}{\mu_{1}-\mu_{2}} \sum_{m=1}^{\infty} \frac{1}{2}\left(\bar{b}_{m}-\mu_{2} \bar{a}_{m}\right) \varsigma_{1}^{-m} \\
& \phi_{2}\left(z_{2}\right)=-\frac{1}{\mu_{1}-\mu_{2}} \sum_{m=1}^{\infty} \frac{1}{2}\left(\bar{b}_{m}-\mu_{1} \bar{a}_{m}\right) \varsigma_{2}^{-m} \\
& \sum_{m=1}^{\infty} \bar{a}_{m} e^{-m \varphi i}=\int\left(-\sigma_{\mathrm{n}} \sin \varphi-\tau \cos \varphi\right) d s \\
& \sum_{m=1}^{\infty} \bar{b}_{m} e^{-m \theta i}=\int\left(\sigma_{n} \cos \varphi-\tau \sin \varphi\right) d s \\
& z_{k}=\frac{1}{2}\left(a-i \mu_{k} b\right) \varsigma_{k}+\frac{1}{2}\left(a+i \mu_{k} b\right) \varsigma_{k}^{-1}
\end{aligned}
$$


where $\zeta_{\mathrm{k}}$ is a complex number that depends on $\mathrm{z}_{\mathrm{k}}$, as shown in the last expression in (16); $\bar{a}_{m}$ and $\bar{b}_{m}$ are the conjugates of the complex numbers $a_{m}$ and $b_{m}$ that are obtained from the normal and shear tractions applied at the boundary of the ellipse, $\sigma_{\mathrm{n}}$ and $\tau$; ds is a differential of the length along the perimeter of the ellipse. See Figure 3. The values of the constants $\mathrm{a}_{\mathrm{m}}$ and $\mathrm{b}_{\mathrm{m}}$ are:

$$
\begin{aligned}
& \overline{\mathrm{a}}_{1}=-\mathrm{a} \sigma_{\mathrm{n}}-\mathrm{ib} \tau \\
& \overline{\mathrm{b}}_{1}=-\mathrm{ib} \sigma_{\mathrm{n}}+\mathrm{a} \tau \\
& \overline{\mathrm{a}}_{\mathrm{m}}=\overline{\mathrm{b}}_{\mathrm{m}}=0, \text { for } \mathrm{m} \geq 2
\end{aligned}
$$

In the vicinity of the tip of the crack, the variable $\mathrm{z}_{\mathrm{k}}$ can be approximated as (Figure 3), $\mathrm{z}_{\mathrm{k}}$ $=\mathrm{a}+\mathrm{r} \cos \theta+\mu_{\mathrm{k}} \mathrm{r} \sin \theta$. Substituting the expression for $\mathrm{z}_{\mathrm{k}}$ and (17) into (16), taking $\mathrm{b}=0$ and eliminating terms larger than $\mathrm{O}\left(\mathrm{r}^{1 / 2}\right)$, one obtains the following expressions for the stress functions:

$$
\begin{aligned}
& \phi_{1}=\frac{\mu_{2} \mathrm{~K}_{\mathrm{I}}+\mathrm{K}_{\mathrm{II}}}{2\left(\mu_{1}-\mu_{2}\right)} \sqrt{\frac{\mathrm{r}}{\pi}}\left(\Psi_{1}-\mathrm{i} \frac{\mu_{12} \sin \theta}{\Psi_{1}}\right) \\
& \phi_{2}=-\frac{\mu_{1} \mathrm{~K}_{\mathrm{I}}+\mathrm{K}_{\mathrm{II}}}{2\left(\mu_{1}-\mu_{2}\right)} \sqrt{\frac{\mathrm{r}}{\pi}\left(\Psi_{2}-\mathrm{i} \frac{\mu_{22} \sin \theta}{\Psi_{2}}\right)} \\
& \Psi_{\mathrm{k}}=\sqrt{\cos \theta+\mu_{\mathrm{k} 1} \sin \theta+\sqrt{\left(\cos \theta+\mu_{\mathrm{k} 1} \sin \theta\right)^{2}+\mu_{\mathrm{k} 2}^{2} \sin ^{2} \theta}} \\
& \mathrm{K}_{\mathrm{I}}=\sigma_{\mathrm{n}} \sqrt{\pi \mathrm{a}} \\
& \mathrm{K}_{\mathrm{II}}=\tau \sqrt{\pi \mathrm{a}}
\end{aligned}
$$

where $\mathrm{K}_{\mathrm{I}}$ and $\mathrm{K}_{\mathrm{II}}$ are the stress intensity factors. Note that they have the same values as those of a crack in an isotropic rock. Taking the derivatives of the stress functions in (18), one obtains: 


$$
\begin{aligned}
& \phi_{1}^{\prime}=-\frac{1}{\sqrt{2 \pi \mathrm{r}}} \frac{\mu_{2} \mathrm{~K}_{\mathrm{I}}+\mathrm{K}_{\mathrm{II}}}{\sqrt{2}\left(\mu_{1}-\mu_{2}\right)} \frac{\Psi_{1}-\mathrm{i} \frac{\mu_{12} \sin \theta}{\Psi_{1}}}{\Psi_{1}^{2}+\frac{\mu_{12}^{2} \sin ^{2} \theta}{\Psi_{1}^{2}}} \\
& \phi_{2}^{\prime}=\frac{1}{\sqrt{2 \pi \mathrm{r}}} \frac{\mu_{1} \mathrm{~K}_{\mathrm{I}}+\mathrm{K}_{\mathrm{II}}}{\sqrt{2}\left(\mu_{1}-\mu_{2}\right)} \frac{\Psi_{2}-\mathrm{i} \frac{\mu_{22} \sin \theta}{\Psi_{2}}}{\Psi_{2}^{2}+\frac{\mu_{22}^{2} \sin ^{2} \theta}{\Psi_{2}^{2}}}
\end{aligned}
$$

Stresses in the coordinate system $\xi-\eta$ are obtained from (19) and (11); note that $F_{0}=0$ in (11). A very important difference between stresses in isotropic rock, equation (1), and in transversely anisotropic rock, equation (19), is that in the former results are independent of the elastic properties of the rock, while in the latter results do depend on elastic properties through the values of the parameters $\mu_{\mathrm{k}}$. Thus, it can be argued, that the SIFs alone, as defined in (19), may not be enough to assess crack initiation in anisotropic rock, as it could be done in isotropic rock, given that they are not sufficient to define unequivocally the stress field at the tip of a crack.

Displacements, also in the $\xi-\eta$ system, are given by (18) and the following expression (20):

$$
\begin{aligned}
& \mathrm{U}_{\xi}=2 \operatorname{Re}\left[\left(\mu_{1}^{2} \mathrm{a}_{11}+\mathrm{a}_{12}-\mu_{1} \mathrm{a}_{13}\right) \phi_{1}\left(\mathrm{z}_{1}\right)+\left(\mu_{2}^{2} \mathrm{a}_{11}+\mathrm{a}_{12}-\mu_{2} \mathrm{a}_{13}\right) \phi_{2}\left(\mathrm{z}_{2}\right)\right] \\
& \mathrm{U}_{\eta}=2 \operatorname{Re}\left[\left(\mu_{1} \mathrm{a}_{12}+\frac{1}{\mu_{1}} \mathrm{a}_{22}-\mathrm{a}_{23}\right) \phi_{1}\left(\mathrm{z}_{1}\right)+\left(\mu_{2} \mathrm{a}_{12}+\frac{1}{\mu_{2}} \mathrm{a}_{22}-\mathrm{a}_{23}\right) \phi_{2}\left(\mathrm{z}_{2}\right)\right]
\end{aligned}
$$

Figure 4 shows the stresses around the tip of a crack obtained from the analytical solution. Results are obtained for the following conditions: isolated crack with length $\mathrm{a}=1 \mathrm{~m}$, in a transversely anisotropic rock with $\mathrm{E}_{\mathrm{x}}=7800 \mathrm{MPa}, \mathrm{E}_{\mathrm{y}}=2400 \mathrm{MPa}, v_{\mathrm{yx}}=0.07, v_{\mathrm{xz}}=0.22$, $v_{\mathrm{yz}}=0.02, \mathrm{G}_{\mathrm{xy}}=830 \mathrm{MPa}$ (the properties are those of the bedded sedimentary Waichecheng series, from Tonon and Amadei, 2002), and subjected to a far-field vertical tensile stress of $1 \mathrm{MPa}$. Two cases are considered. The first one of a crack parallel to the x-axis, i.e. $\beta=0$ (see Figure 2), and the second one of a crack at $45^{\circ}$ with the axis of elastic symmetry, i.e. $\beta=45^{\circ}$. Note that the first case, $\beta=0$, corresponds to pure mode I 
loading. Also, for the crack at $\beta=45^{\circ}$, stresses in Figure 4 are rotated $45^{\circ}$ for better visualization and comparison of the results. Figure 4 also includes results obtained with the code ABAQUS (ABAQUS, 2013) with the same crack geometry and rock properties. ABAQUS is a general purpose Finite Element Method code that has been extensively used and validated in geomechanics, in general, and in fracture mechanics in particular (e.g. Bobet and Einstein, 1998; Bobet, 2001; Bobet et al. 2009). The mesh used for the ABAQUS simulations consists of regular (i.e. nodes at the corner and center of element sides) eight-node quadrilateral isoparametric elements, except the elements around the tip of the crack that have quarter-node elements to simulate better the square-root singularity. As one can see, the comparison is excellent. As expected, large concentrations of stresses occur at the tip, with singularities present for all stresses. Because of the tensile loading, both $\sigma_{\mathrm{xx}}$ and $\sigma_{\mathrm{xy}}$ are tensile ahead of the tip.

The displacements, given by equation (20), can be used to backcalculate, from a numerical method, the SIFs at the tip of a crack. By using $\theta=\pi$ in (18), that is by obtaining the displacements of the crack behind the tip, the following expressions for the SIFs can be used:

$$
\begin{aligned}
& \mathrm{K}_{\mathrm{I}}=\frac{\sqrt{2 \pi}}{2 \sqrt{\mathrm{r}}} \frac{\mathrm{a}_{22} \mathrm{~B}_{\mathrm{II}} \mathrm{U}_{\xi}^{\pi}-\mathrm{a}_{11} \mathrm{~A}_{\mathrm{II}} \mathrm{U}_{\eta}^{\pi}}{\mathrm{a}_{11} \mathrm{a}_{22}\left(\mathrm{~A}_{\mathrm{I}} \mathrm{B}_{\mathrm{II}}-\mathrm{B}_{\mathrm{I}} \mathrm{A}_{\text {II }}\right)} \\
& \mathrm{K}_{\mathrm{II}}=-\frac{\sqrt{2 \pi}}{2 \sqrt{\mathrm{r}}} \frac{\mathrm{a}_{22} \mathrm{~B}_{\mathrm{I}} \mathrm{U}_{\xi}^{\pi}-\mathrm{a}_{11} \mathrm{~A}_{\mathrm{I}} \mathrm{U}_{\eta}^{\pi}}{\mathrm{a}_{11} \mathrm{a}_{22}\left(\mathrm{~A}_{\mathrm{I}} \mathrm{B}_{\mathrm{II}}-\mathrm{B}_{\mathrm{I}} \mathrm{A}_{\mathrm{II}}\right)} \\
& \mathrm{A}_{\mathrm{I}}=\mu_{11} \mu_{22}+\mu_{21} \mu_{12} \\
& \mathrm{~A}_{\mathrm{II}}=\mu_{12}+\mu_{22} \\
& \mathrm{~B}_{\mathrm{I}}=\frac{\mu_{12}}{\mu_{11}^{2}+\mu_{12}^{2}}+\frac{\mu_{22}}{\mu_{21}^{2}+\mu_{22}^{2}} \\
& \mathrm{~B}_{\mathrm{II}}=\frac{\mu_{11} \mu_{22}+\mu_{21} \mu_{12}}{\left(\mu_{11}^{2}+\mu_{12}^{2}\right)\left(\mu_{21}^{2}+\mu_{22}^{2}\right)}
\end{aligned}
$$

where $U_{\xi}^{\pi}$ and $U_{\eta}^{\pi}$ are the values of the displacements computed at $\theta=\pi$.

Figure 5 shows the results of using equations (21) and the code FROCK (Bobet and Garcia Marin, 2013). The problem addressed is the same as that of Figure 4, except that 
the crack length is $a=0.5$ and the angle $\beta$ ranges from 0 to $90^{\circ}$. The FROCK results are compared with the theoretical values of $\mathrm{K}_{\mathrm{I}}=\sigma \sqrt{\pi \mathrm{a}} \cos ^{2} \beta$ and $\mathrm{K}_{\mathrm{II}}=\sigma \sqrt{\pi \mathrm{a}} \sin \beta \cos \beta$. The comparisons are excellent.

\section{Crack in saturated transversely anisotropic elastic rock with undrained loading}

When a rock is saturated and is subjected to changes of stresses, excess pore pressures may be induced. With time, the excess pore pressures dissipate and a steady-state condition is attained. However, at the limit, when the rate of loading is much faster than the capacity of the rock to dissipate excess pore pressures, undrained conditions apply. This situation may occur when the load is imposed very quickly, e.g. hydraulic fracturing, and the permeability of the rock is very low, e.g. a shale. It is thus interesting to investigate how undrained conditions affect the stress field at the tip of a crack under mixed mode I-II loading.

Undrained conditions are attained when no change of fluid volume in the rock occurs. In other words, when $\chi=0$. From equations (5) and (2),

$$
\begin{aligned}
& \Delta \mathrm{u}=-\frac{1}{\beta_{3}}\left(\beta_{1} \sigma_{\mathrm{xx}}+\beta_{2} \sigma_{y y}\right) \\
& \beta_{3}=\frac{1}{M}+\alpha_{x} \beta_{1}+\alpha_{y} \beta_{2}
\end{aligned}
$$

where $\Delta \mathrm{u}$ are the excess pore pressures. Given equation (2) and the expression for excess pore pressures in (22), strains in the $x-y$ coordinate system are:

$$
\begin{aligned}
& \varepsilon_{\mathrm{xx}}=\left(\alpha_{1}-\frac{\beta_{1}^{2}}{\beta_{3}}\right) \sigma_{\mathrm{xx}}-\left(\alpha_{2}+\frac{\beta_{1} \beta_{2}}{\beta_{3}}\right) \sigma_{\mathrm{yy}}=\mathrm{A}_{1} \sigma_{\mathrm{xx}}-\mathrm{A}_{2} \sigma_{\mathrm{yy}} \\
& \varepsilon_{\mathrm{yy}}=-\left(\alpha_{2}+\frac{\beta_{1} \beta_{2}}{\beta_{3}}\right) \sigma_{\mathrm{xx}}+\left(\alpha_{3}-\frac{\beta_{2}^{2}}{\beta_{3}}\right) \sigma_{\mathrm{yy}}=-\mathrm{A}_{2} \sigma_{\mathrm{xx}}+\mathrm{A}_{3} \sigma_{\mathrm{yy}} \\
& \gamma_{\mathrm{xy}}=\frac{\tau_{\mathrm{xy}}}{\mathrm{G}_{\mathrm{xy}}}
\end{aligned}
$$


In the $\xi-\eta$ coordinate system, strains are:

$$
\begin{aligned}
& \varepsilon_{\xi \xi}=a_{11} \sigma_{\xi \xi}+a_{12} \sigma_{\eta \eta}+a_{13} \sigma_{\xi \eta} \\
& \varepsilon_{\eta \eta}=a_{21} \sigma_{\xi \xi}+a_{22} \sigma_{\eta \eta}+a_{23} \sigma_{\xi \eta} \\
& \gamma_{\xi \eta}=a_{31} \sigma_{\xi \xi}+a_{32} \sigma_{\eta \eta}+a_{33} \sigma_{\xi \eta} \\
& a_{11}=A_{1} \cos ^{4} \beta+A_{3} \sin ^{4} \beta+\left(\frac{1}{G_{x y}}-2 A_{2}\right) \sin ^{2} \beta \cos ^{2} \beta \\
& a_{12}=a_{21}=-\left[A_{2}\left(\cos ^{4} \beta+\sin ^{4} \beta\right)+\left(\frac{1}{G_{x y}}-A_{1}-A_{3}\right) \sin ^{2} \beta \cos ^{2} \beta\right] \\
& a_{13}=a_{31}=\left[\left(\frac{1}{G_{x y}}-2 A_{1}\right) \cos ^{2} \beta-\left(\frac{1}{G_{x y}}-2 A_{3}\right) \sin ^{2} \beta-2 A_{2} \cos 2 \beta\right] \sin \beta \cos \beta \\
& a_{22}=A_{3} \cos ^{4} \beta+A_{1} \sin ^{4} \beta+\left(\frac{1}{G_{x y}}-2 A_{2}\right) \sin ^{2} \beta \cos ^{2} \beta \\
& a_{23}=a_{32}=\left[\left(\frac{1}{G_{x y}}-2 A_{1}\right) \sin ^{2} \beta-\left(\frac{1}{G_{x y}}-2 A_{3}\right) \cos ^{2} \beta+2 A_{2} \cos 2 \beta\right] \sin \beta \cos \beta \\
& a_{33}=\left(A_{1}+2 A_{2}+A_{3}\right) \sin ^{2} 2 \beta+\frac{1}{G_{x y}} \cos ^{2} 2 \beta
\end{aligned}
$$

where $A_{1}, A_{2}$ and $A_{3}$ are defined in (23).

Equation (12) is still the characteristic equation. It has two roots that, similar to the case of dry ground, are the complex numbers $\mu_{\mathrm{k}}$; the $\mu_{\mathrm{k}}$ values can also be obtained from equation (13) using the constants $\mathrm{a}_{11}, \mathrm{a}_{12}$, etc. given in (24). In (13), $\mu_{\mathrm{k}}^{\mathrm{o}}$, and its conjugate $\bar{\mu}_{\mathrm{k}}^{\mathrm{o}}$, are the roots of the characteristic equation in the $\mathrm{x}-\mathrm{y}$ coordinate system that is given by:

$A_{1} \mu_{k}^{4}+\left(\frac{1}{G_{x y}}-2 A_{2}\right) \mu_{k}^{2}+A_{3}=0$

Effective stresses and excess pore pressures, in the $\xi$ - $\eta$ coordinate system are: 


$$
\begin{aligned}
& \sigma_{\text {s. }}^{\prime}=2 \operatorname{Re}\left\{\left[\mu_{1}^{2}-\frac{1}{\beta_{3}}\left(\alpha_{x} \cos ^{2} \beta+\alpha_{y} \sin ^{2} \beta\right)\left[\beta_{1}\left(\sin \beta+\mu_{1} \cos \beta\right)^{2}+\beta_{2}\left(\cos \beta-\mu_{1} \sin \beta\right)^{2}\right]\right] \phi_{1}^{\prime}\left(z_{1}\right)+\right. \\
& \left.\left[\mu_{2}^{2}-\frac{1}{\beta_{3}}\left(\alpha_{x} \cos ^{2} \beta+\alpha_{y} \sin ^{2} \beta\right)\left[\beta_{1}\left(\sin \beta+\mu_{2} \cos \beta\right)^{2}+\beta_{2}\left(\cos \beta-\mu_{2} \sin \beta\right)^{2}\right]\right] \phi_{2}^{\prime}\left(z_{2}\right)\right\} \\
& \sigma_{\eta 11}^{\prime}=2 \operatorname{Re}\left\{\left[1-\frac{1}{\beta_{3}}\left(\alpha_{x} \sin ^{2} \beta+\alpha_{y} \cos ^{2} \beta\right)\left[\beta_{1}\left(\sin \beta+\mu_{1} \cos \beta\right)^{2}+\beta_{2}\left(\cos \beta-\mu_{1} \sin \beta\right)^{2}\right]\right] \phi_{1}^{\prime}\left(z_{1}\right)+\right. \\
& \left.\left[1-\frac{1}{\beta_{3}}\left(\alpha_{x} \sin ^{2} \beta+\alpha_{y} \cos ^{2} \beta\right)\left[\beta_{1}\left(\sin \beta+\mu_{2} \cos \beta\right)^{2}+\beta_{2}\left(\cos \beta-\mu_{2} \sin \beta\right)^{2}\right]\right] \phi_{2}^{\prime}\left(z_{2}\right)\right\} \\
& \tau_{y_{1}}=-2 \operatorname{Re}\left[\mu_{1} \phi_{1}^{\prime}\left(z_{1}\right)+\mu_{2} \phi_{2}^{\prime}\left(z_{2}\right)\right] \\
& \Delta \mathrm{u}=-\frac{2}{\beta_{3}} \operatorname{Re}\left\{\left[\beta_{1}\left(\sin \beta+\mu_{1} \cos \beta\right)^{2}+\beta_{2}\left(\cos \beta-\mu_{1} \sin \beta\right)^{2}\right] \phi_{1}^{\prime}\left(x_{1}\right)+\right. \\
& \left.\left[\beta_{1}\left(\sin \beta+\mu_{2} \cos \beta\right)^{2}+\beta_{2}\left(\cos \beta-\mu_{2} \sin \beta\right)^{2}\right] \phi_{2}^{\prime}\left(z_{2}\right)\right\}
\end{aligned}
$$

where $\sigma^{\prime} \xi \xi$ and $\sigma_{\eta \eta}^{\prime}$ are the effectives stress, which are related to pore pressures and total stresses by Equation (15). The stress functions $\phi_{1}$ and $\phi_{2}$, and $\phi^{\prime}{ }_{1}$ and $\phi_{2}^{\prime}$, are given by (18) and (19), respectively, using the values for $\mu_{\mathrm{k}}$ given by (13) with (25). Analogously, displacements are obtained with (20) using the constants given in (24).

The solution obtained for undrained loading has been verified by providing comparisons with results obtained from the FEM ABAQUS. Figures 6 and 7 include contour plots of the effective, shear stresses and excess pore pressures in the vicinity of a crack. The same geometry, loading and material properties as those used for Figure 4 are employed, with $\alpha_{x}=\alpha_{y}=1$ and $M>>1$. Figure 6 provides results for a horizontal crack, $\beta=0$, and Figure 7 for a crack at $45^{\circ}, \beta=45^{\circ}$. As one can see the differences are acceptable. What is interesting to note is the large magnitude of negative excess pore pressures at the tip, which are consistent with the tensile load imposed to the medium. A comparison between stresses in Figure 6 and Figure 4(a), (c) and (e) and between Figure 7 and Figure 4(b), (d) and (f) indicates that undrained loading results in effective and shear stresses that have maxima at different directions than for dry loading. Also, stresses are much smaller in the undrained loading than in the dry loading; some of the stresses even become negative. This is the result of the development of large excess pore pressures to prevent any change of volume in the rock, which in turn reduce the effective stresses. Further discussion is provided later in the paper. 


\section{Crack in saturated transversely anisotropic elastic rock with drainage}

When the rock is saturated and has a large permeability or after a long period of time injecting fluid into a fracture, steady-state conditions are obtained. In this situation, a steady flow of fluid occurs in the vicinity of the crack that changes the stresses around the tip. The solution of Equation (4), without the transient term on the right hand side of the expression for steady-state conditions, provides the distribution of the pore pressures inside the rock. It is convenient to express Equation (4) in terms of the $\xi-\eta$ coordinate system shown in Figure 2. The equation takes the form:

$\mathrm{k}_{\xi \xi} \frac{\partial^{2} \mathrm{u}}{\partial \xi^{2}}+2 \mathrm{k}_{\xi \eta} \frac{\partial^{2} \mathrm{u}}{\partial \xi \partial \eta}+\mathrm{k}_{\eta \eta} \frac{\partial^{2} \mathrm{u}}{\partial \eta^{2}}=0$

where $\mathrm{k}_{\xi \xi}, \mathrm{k}_{\xi \eta}$ and $\mathrm{k}_{\xi \eta}$ are the permeabilities in the $\xi-\eta$ coordinates. The equation can be solved introducing the complex variable $\mathrm{z}_{3}=\xi+\mu_{3} \eta$ where $\mu_{3}$ is a complex number that has the following value:

$\mu_{3}=\frac{\left(k_{x}-k_{y}\right) \sin \beta \cos \beta \pm i \sqrt{k_{x} k_{y}}}{k_{x} \sin ^{2} \beta+k_{y} \cos ^{2} \beta}$

The pore pressure distribution around an elliptical hole (see Figure 2) with constant pressure $\mathrm{u}_{\mathrm{o}}$ at the perimeter of the ellipse and free field pressure $\mathrm{u}_{\mathrm{ff}}$ is given by the following:

$\mathrm{u}=\mathrm{u}_{\mathrm{ff}}-\frac{\mathrm{u}_{\mathrm{o}}-\mathrm{u}_{\mathrm{ff}}}{\ln \mathrm{R}} \operatorname{Re}\left[\ln \frac{\mathrm{S}_{3}}{\mathrm{R}}\right]$

where $\mathrm{R}$ is the distance from the opening where far field pore pressures are restored, and $\zeta_{3}$ is the complex variable that conformally maps the elliptical opening into a circular opening. It is related to the complex variable $\mathrm{z}_{3}$, as follows:

$z_{3}=\xi+\mu_{3} \eta=\frac{1}{2}\left(a-i \mu_{3} b\right) \varsigma_{3}+\frac{1}{2}\left(a+i \mu_{3} b\right) \frac{1}{\varsigma_{3}}$ 
From (29), one can obtain the flow in or out of the opening, as well as the magnitude of the seepage flow inside the rock. They are given by the following expressions:

$$
\begin{aligned}
& \mathrm{Q}=2 \pi \frac{\mathrm{u}_{\mathrm{o}}-\mathrm{u}_{\mathrm{ff}}}{\gamma_{\mathrm{w}} \ln \mathrm{R}} \operatorname{Re}\left[\frac{\left(\mathrm{k}_{\xi \xi}+\mu_{3} \mathrm{k}_{\xi \eta}\right) \mathrm{b}-\mathrm{i}\left(\mathrm{k}_{\xi \eta}+\mu_{3} \mathrm{k}_{\eta \eta}\right) \mathrm{a}}{\mathrm{a}-\mathrm{i} \mu_{3} \mathrm{~b}}\right] \\
& \mathrm{V}_{\xi}=2 \frac{\mathrm{u}_{\mathrm{o}}-\mathrm{u}_{\mathrm{ff}}}{\gamma_{\mathrm{w}} \ln \mathrm{R}} \operatorname{Re}\left[\frac{\left(\mathrm{k}_{\xi \xi}+\mu_{3} \mathrm{k}_{\xi \eta}\right) \varsigma_{3}}{\left(\mathrm{a}-\mathrm{i} \mu_{3} \mathrm{~b}\right) \varsigma_{3}^{2}-\left(\mathrm{a}+\mathrm{i} \mu_{3} \mathrm{~b}\right)}\right] \\
& \mathrm{V}_{\eta}=2 \frac{\mathrm{u}_{\mathrm{o}}-\mathrm{u}_{\mathrm{ff}}}{\gamma_{\mathrm{w}} \ln \mathrm{R}} \operatorname{Re}\left[\frac{\left(\mathrm{k}_{\xi \eta}+\mu_{3} \mathrm{k}_{\eta \eta}\right) \varsigma_{3}}{\left(\mathrm{a}-\mathrm{i} \mu_{3} \mathrm{~b}\right) \varsigma_{3}^{2}-\left(\mathrm{a}+\mathrm{i} \mu_{3} \mathrm{~b}\right)}\right]
\end{aligned}
$$

where $Q$ is the flow, $V_{\xi}$ and $V_{\eta}$ are the seepage velocities along the $\xi$ and $\eta$ directions, respectively, and $\gamma_{\mathrm{w}}$ is the unit weight of the fluid. Note that the expressions for a crack are obtained by taking $b=0$.

Figure 2 depicts the problem to be solved: an isolated crack in a transversely elastic anisotropic medium, inclined at an angle $\beta$ with the global coordinate system $x-y$, which corresponds to the axes of anisotropic symmetry, and subjected to internal fluid pressure $\mathrm{u}_{\mathrm{o}}$, far-field fluid pressure $\mathrm{u}_{\mathrm{ff}}$, and no tractions applied either at the surfaces of the crack or at the far-field boundaries. That is, the crack is stress free. The problem is decomposed into two: one where the inside of the crack is subjected to constant fluid pressure $\mathrm{u}_{\mathrm{ff}}$ and the far field is also subjected to a constant fluid pressure $\mathrm{u}_{\mathrm{ff}}$; and two, where the inside crack has a constant fluid pressure $\mathrm{u}_{0}-\mathrm{u}_{\mathrm{ff}}$ and the far field has zero fluid pressure. The superposition of the two solutions provides the complete answer. However, problem one has the trivial solution of constant total stresses and pore pressures equal to $\mathrm{u}_{\mathrm{ff}}$ and thus it does not create a singularity at the tip of the crack.

The solution of the second problem must satisfy Equation (9), the compatibility equation, and the boundary conditions. A particular solution of Equation (9) is:

$$
\begin{aligned}
& F_{o}^{\prime \prime}=\frac{d^{2} F}{d z_{3}^{2}}=\frac{u_{o}-u_{\text {ff }}}{\ln R} \operatorname{Re}\left[N \ln \frac{\varsigma_{3}}{R}\right] \\
& N=\frac{\beta_{1}\left(\sin \beta+\mu_{3} \cos \beta\right)^{2}+\beta_{2}\left(\cos \beta-\mu_{3} \sin \beta\right)^{2}}{a_{11} \mu_{3}^{4}-2 a_{13} \mu_{3}^{3}+\left(a_{33}+2 a_{12}\right) \mu_{3}^{2}-2 a_{23} \mu_{3}+a_{22}}
\end{aligned}
$$


where the constants $a_{11}, a_{12}$, etc. are given in (7). The stresses associated with the particular solution induce displacements that are not unique, i.e. displacements are not univocally defined at, say, $\eta^{+}=0$ (approaching from the positive $\eta$ axis) and $\eta^{-}=0$ (approaching from the negative $\eta$ axis). A suitable solution is found by dividing the continuum into two half spaces: one for $\eta \geq 0$ and the other for $\eta \leq 0$, and then imposing compatibility of stresses and displacements at the common boundary $\eta=0$. This is a similar approach taken by Atkinson and Clements (1977) and Clements (1973), to find thermally-induced stresses at the tip of a crack in an anisotropic medium, and also by Aköz and Tauchert (1972) to find thermal stresses in an orthotropic elastic semi-space. We try the following stress functions:

$$
\begin{aligned}
& \phi_{1}^{\prime}=\frac{1}{2} \frac{\mathrm{u}_{\mathrm{o}}-\mathrm{u}_{\mathrm{ff}}}{\ln \mathrm{R}} \mathrm{A}_{1} \ln \frac{\varsigma_{1}}{\mathrm{R}} \\
& \phi_{2}^{\prime}=\frac{1}{2} \frac{\mathrm{u}_{\mathrm{o}}-\mathrm{u}_{\mathrm{ff}}}{\ln \mathrm{R}} \mathrm{A}_{2} \ln \frac{\varsigma_{2}}{\mathrm{R}}
\end{aligned}
$$

where $A_{1}$ and $A_{2}$ are complex constants and

$$
\mathrm{z}_{\mathrm{k}}=\xi+\mu_{\mathrm{k}} \eta=\frac{1}{2} \mathrm{a}\left(\varsigma_{\mathrm{k}}+\varsigma_{\mathrm{k}}^{-1}\right) \quad \mathrm{k}=1,2
$$

and $\mu_{\mathrm{k}}$ are the complex roots of Equation (12), which are given in Equation (13). From (32) and (33), stresses and displacements for the upper half space are: 


$$
\begin{aligned}
& \sigma_{\xi \xi}^{+}=\frac{\mathrm{u}_{\mathrm{o}}-\mathrm{u}_{\mathrm{ff}}}{\ln \mathrm{R}} \operatorname{Re}\left[\mathrm{A}_{1}^{+} \mu_{1}^{2} \ln \frac{\varsigma_{1}}{\mathrm{R}}+\mathrm{A}_{2}^{+} \mu_{2}^{2} \ln \frac{\varsigma_{2}}{\mathrm{R}}+\mathrm{N} \mu_{3}^{2} \ln \frac{\varsigma_{3}}{\mathrm{R}}\right] \\
& \sigma_{\eta \eta}^{+}=\frac{\mathrm{u}_{\mathrm{o}}-\mathrm{u}_{\mathrm{ff}}}{\ln \mathrm{R}} \operatorname{Re}\left[\mathrm{A}_{1}^{+} \ln \frac{\varsigma_{1}}{\mathrm{R}}+\mathrm{A}_{2}^{+} \ln \frac{\varsigma_{2}}{\mathrm{R}}+\mathrm{N} \ln \frac{\varsigma_{3}}{\mathrm{R}}\right] \\
& \sigma_{\xi \eta}^{+}=-\frac{\mathrm{u}_{\mathrm{o}}-\mathrm{u}_{\mathrm{ff}}}{\ln \mathrm{R}} \operatorname{Re}\left[\mathrm{A}_{1}^{+} \mu_{1} \ln \frac{\varsigma_{1}}{\mathrm{R}}+\mathrm{A}_{2}^{+} \mu_{2} \ln \frac{\varsigma_{2}}{\mathrm{R}}+\mathrm{N} \mu_{3} \ln \frac{\varsigma_{3}}{\mathrm{R}}\right] \\
& \mathrm{U}_{\xi}^{+}=\frac{\mathrm{a}}{2} \frac{\mathrm{u}_{\mathrm{o}}-\mathrm{u}_{\mathrm{ff}}}{\ln \mathrm{R}} \operatorname{Re}\left[\begin{array}{l}
\mathrm{A}_{\xi 1} \mathrm{~A}_{1}^{+}\left(\frac{1-\varsigma_{1}^{2}}{\varsigma_{1}}+\frac{1+\varsigma_{1}^{2}}{\varsigma_{1}} \ln \frac{\varsigma_{1}}{\mathrm{R}}\right)+\mathrm{A}_{\xi_{2}} \mathrm{~A}_{2}^{+}\left(\frac{1-\varsigma_{2}^{2}}{\varsigma_{2}}+\frac{1+\varsigma_{2}^{2}}{\varsigma_{2}} \ln \frac{\varsigma_{2}}{\mathrm{R}}\right)+ \\
{\left[\mathrm{A}_{\xi 3} \mathrm{~N}-\left(\beta_{1} \cos ^{2} \beta+\beta_{2} \sin ^{2} \beta\right)\right]\left(\frac{1-\varsigma_{3}^{2}}{\varsigma_{3}}+\frac{1+\varsigma_{3}^{2}}{\varsigma_{3}} \ln \frac{\varsigma_{3}}{\mathrm{R}}\right)}
\end{array}\right]
\end{aligned}
$$

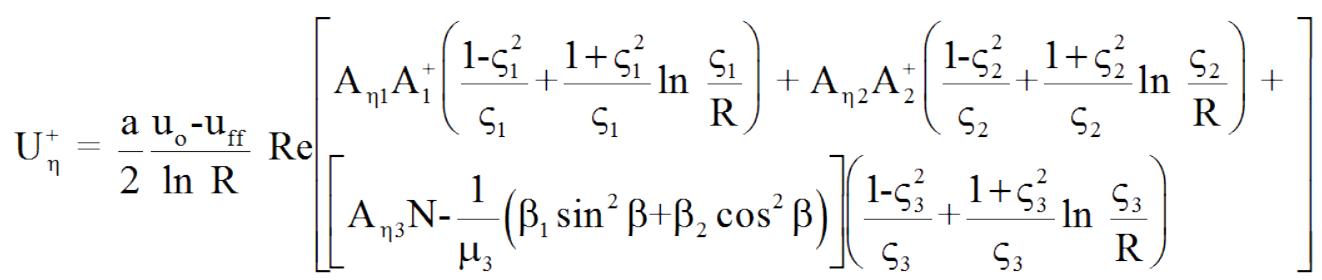

and for the lower half,

$$
\begin{aligned}
& \sigma_{\xi \xi}^{-}=-\frac{\mathrm{u}_{\mathrm{o}}-\mathrm{u}_{\mathrm{ff}}}{\ln \mathrm{R}} \operatorname{Re}\left[\mathrm{A}_{1}^{-} \mu_{1}^{2} \ln \frac{-\varsigma_{1}}{\mathrm{R}}+\mathrm{A}_{2}^{-} \mu_{2}^{2} \ln \frac{-\varsigma_{2}}{\mathrm{R}}-\mathrm{N} \mu_{3}^{2} \ln \frac{-\varsigma_{3}}{\mathrm{R}}\right] \\
& \sigma_{\eta \eta}^{-}=-\frac{\mathrm{u}_{\mathrm{o}}-\mathrm{u}_{\mathrm{ff}}}{\ln \mathrm{R}} \operatorname{Re}\left[\mathrm{A}_{1}^{-} \ln \frac{-\varsigma_{1}}{\mathrm{R}}+\mathrm{A}_{2}^{-} \ln \frac{-\varsigma_{2}}{\mathrm{R}}-\mathrm{N} \ln \frac{-\varsigma_{3}}{\mathrm{R}}\right] \\
& \sigma_{\xi \eta}^{-}=\frac{\mathrm{u}_{\mathrm{o}}-\mathrm{u}_{\mathrm{ff}}}{\ln \mathrm{R}} \operatorname{Re}\left[\mathrm{A}_{1}^{-} \mu_{1} \ln \frac{-\varsigma_{1}}{\mathrm{R}}+\mathrm{A}_{2}^{-} \mu_{2} \ln \frac{-\varsigma_{2}}{\mathrm{R}}-\mathrm{N} \mu_{3} \ln \frac{-\varsigma_{3}}{\mathrm{R}}\right] \\
& \mathrm{U}_{\xi}^{-}=-\frac{\mathrm{a}}{2} \frac{\mathrm{u}_{\mathrm{o}}-\mathrm{u}_{\mathrm{ff}}}{\ln \mathrm{R}} \mathrm{Re}\left[\begin{array}{l}
\mathrm{A}_{\xi_{1}} \mathrm{~A}_{1}^{-}\left(\frac{1-\varsigma_{1}^{2}}{\varsigma_{1}}+\frac{1+\varsigma_{1}^{2}}{\varsigma_{1}} \ln \frac{-\varsigma_{1}}{\mathrm{R}}\right)+\mathrm{A}_{\xi_{2}} \mathrm{~A}_{2}^{-}\left(\frac{1-\varsigma_{2}^{2}}{\varsigma_{2}}+\frac{1+\varsigma_{2}^{2}}{\varsigma_{2}} \ln \frac{-\varsigma_{2}}{\mathrm{R}}\right)- \\
{\left[\mathrm{A}_{\xi 3} \mathrm{~N}-\left(\beta_{1} \cos ^{2} \beta+\beta_{2} \sin ^{2} \beta\right)\right]\left(\frac{1-\varsigma_{3}^{2}}{\varsigma_{3}}+\frac{1+\varsigma_{3}^{2}}{\varsigma_{3}} \ln \frac{-\varsigma_{3}}{\mathrm{R}}\right)}
\end{array}\right]
\end{aligned}
$$

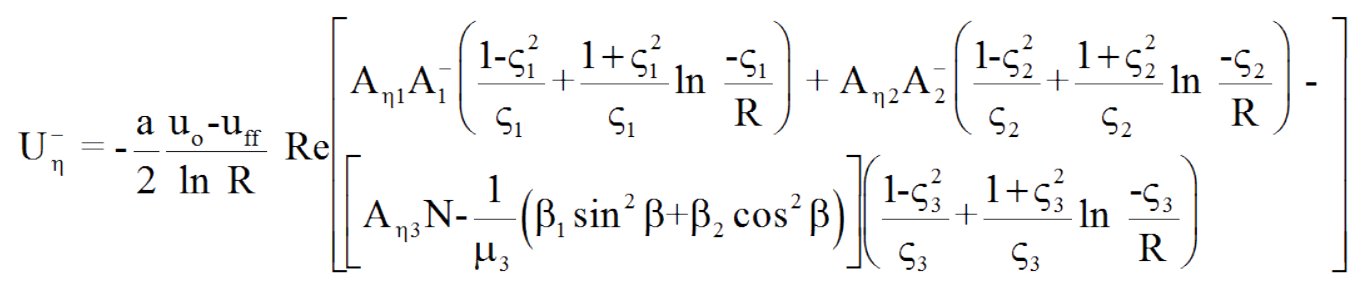

where 


$$
\begin{aligned}
& \mathrm{A}_{\xi \mathrm{k}}=\mu_{\mathrm{k}}^{2} \mathrm{a}_{11}+\mathrm{a}_{12}-\mu_{\mathrm{k}} \mathrm{a}_{13} \\
& \mathrm{~A}_{\eta \mathrm{k}}=\mu_{\mathrm{k}} \mathrm{a}_{12}+\frac{1}{\mu_{\mathrm{k}}} \mathrm{a}_{22}-\mathrm{a}_{23}
\end{aligned}
$$

The constants $\mathrm{A}_{1}^{+}, \mathrm{A}_{2}^{+}, \mathrm{A}_{1}^{-}$and $\mathrm{A}_{2}^{-}$are found imposing compatibility of displacements and stresses at the common boundary, i.e. at $\eta=0$. Thus,

$$
\begin{aligned}
& \left.\sigma_{\eta \eta}^{+}\right|_{\eta=0}=\left.\sigma_{\eta \eta}^{-}\right|_{\eta=0} \\
& \left.\sigma_{\xi \eta}^{+}\right|_{\eta=0}=\left.\sigma_{\xi \eta}^{-}\right|_{\eta=0} \\
& \left.U_{\xi}^{+}\right|_{\eta=0}=\left.U_{\xi}^{-}\right|_{\eta=0} \\
& \left.U_{\eta}^{+}\right|_{\eta=0}=\left.U_{\eta}^{-}\right|_{\eta=0}
\end{aligned}
$$

The constants are obtained from the following system of six linear equations,

$$
\begin{aligned}
& \mathrm{A}_{12}^{+}+\mathrm{A}_{22}^{+}=-\mathrm{N}_{2} \\
& \mu_{12} \mathrm{~A}_{11}^{+}+\mu_{11} \mathrm{~A}_{12}^{+}+\mu_{22} \mathrm{~A}_{21}^{+}+\mu_{21} \mathrm{~A}_{22}^{+}=-\mu_{32} \mathrm{~N}_{1}-\mu_{31} \mathrm{~N}_{2} \\
& \mathrm{~A}_{\xi 12} \mathrm{~A}_{11}^{+}+\mathrm{A}_{\xi 11} \mathrm{~A}_{12}^{+}+\mathrm{A}_{\xi 22} \mathrm{~A}_{21}^{+}+\mathrm{A}_{\xi 21} \mathrm{~A}_{22}^{+}=-\mathrm{A}_{\xi 32} \mathrm{~N}_{1}-\mathrm{A}_{\xi 31} \mathrm{~N}_{2} \\
& \mathrm{~A}_{\eta 12} \mathrm{~A}_{11}^{+}+\mathrm{A}_{\eta 11} \mathrm{~A}_{12}^{+}+\mathrm{A}_{\eta 22} \mathrm{~A}_{21}^{+}+\mathrm{A}_{\eta 21} \mathrm{~A}_{22}^{+}=-\mathrm{A}_{\eta 32} \mathrm{~N}_{1}-\mathrm{A}_{\eta 31} \mathrm{~N}_{2}-\left(\beta_{1} \sin ^{2} \beta+\beta_{2} \cos ^{2} \beta\right) \frac{\mu_{32}}{\mu_{31}^{2}+\mu_{32}^{2}} \\
& \mathrm{~A}_{1}^{+}=-\mathrm{A}_{1}^{-} \\
& \mathrm{A}_{2}^{+}=-\mathrm{A}_{2}^{-}
\end{aligned}
$$

where the following notation has been used for any complex variable: $\Omega_{\mathrm{i}}=\Omega_{\mathrm{i} 1}+\mathrm{i} \Omega_{\mathrm{i} 2}$, where $\Omega_{\mathrm{i} 1}$ and $\Omega_{\mathrm{i} 2}$ are real numbers, e.g. from equation (32), $\mathrm{N}=\mathrm{N}_{1}+\mathrm{i} \mathrm{N}_{2}$.

The stresses given by equations (35) and (36) satisfy the boundary conditions of zero stresses far from the crack, i.e. at $\zeta=\mathrm{R}$, provided that $\mathrm{R}>\mathrm{a}$, but introduce non-zero normal and shear stresses at the crack surfaces. New stress functions are added to eliminate the stresses at the crack surface, while not changing the far-field stresses. These functions are: 


$$
\begin{aligned}
& \phi_{1}=-\frac{B_{2}+\mu_{2} B_{1}}{2\left(\mu_{1}-\mu_{2}\right)} \sqrt{\operatorname{ar}}\left(\Psi_{1}-i \frac{\mu_{12} \sin \theta}{\Psi_{1}}\right) \\
& \phi_{2}=\frac{\mathrm{B}_{2}+\mu_{1} \mathrm{~B}_{1}}{2\left(\mu_{1}-\mu_{2}\right)} \sqrt{\mathrm{ar}}\left(\Psi_{2}-\mathrm{i} \frac{\mu_{22} \sin \theta}{\Psi_{2}}\right) \\
& \phi_{1}^{\prime}=\frac{B_{2}+\mu_{2} B_{1}}{2\left(\mu_{1}-\mu_{2}\right)} \sqrt{\frac{a}{r}} \frac{\Psi_{1}-i \frac{\mu_{12} \sin \theta}{\Psi_{1}}}{\Psi_{1}^{2}+\frac{\mu_{12}^{2} \sin ^{2} \theta}{\Psi_{1}^{2}}} \\
& \phi_{2}^{\prime}=-\frac{B_{2}+\mu_{1} B_{1}}{2\left(\mu_{1}-\mu_{2}\right)} \sqrt{\frac{a}{r}} \frac{\Psi_{2}-i \frac{\mu_{22} \sin \theta}{\Psi_{2}}}{\Psi_{2}^{2}+\frac{\mu_{22}^{2} \sin ^{2} \theta}{\Psi_{2}^{2}}} \\
& \Psi_{\mathrm{k}}=\sqrt{\cos \theta+\mu_{\mathrm{k} 1} \sin \theta+\sqrt{\left(\cos \theta+\mu_{\mathrm{k} 1} \sin \theta\right)^{2}+\mu_{\mathrm{k} 2}^{2} \sin ^{2} \theta}} \\
& \mathrm{B}_{1}=\left(\mathrm{u}_{\mathrm{o}}-\mathrm{u}_{\mathrm{ff}}\right) \operatorname{Re}\left[\mathrm{A}_{1}^{+}+\mathrm{A}_{2}^{+}+\mathrm{N}\right] \\
& \mathrm{B}_{2}=-\left(\mathrm{u}_{\mathrm{o}}-\mathrm{u}_{\mathrm{ff}}\right) \operatorname{Re}\left[\mu_{1} \mathrm{~A}_{1}^{+}+\mu_{2} \mathrm{~A}_{2}^{+}+\mu_{3} \mathrm{~N}\right]
\end{aligned}
$$

The stresses in Equations (35) and (36) are not singular at the tip. As a result, the stresses around the tip of the crack are dominated by those given by the stress functions in (40). The pore pressures, given by (29), are also not singular, and thus near the tip total stresses and effective stresses have the same magnitude. Stresses, i.e. total or effective, can then be computed using equations (11), (20) and (40). Note that inspection of the expressions for $\phi_{1}$ and $\phi_{2}$, or $\phi^{\prime}{ }_{1}$ and $\phi^{\prime}{ }_{2}$, in (40) suggest that $\mathrm{B}_{1}$ and $\mathrm{B}_{2}$ play a role similar to that of $\mathrm{K}_{\mathrm{I}}$ and $\mathrm{K}_{\mathrm{II}}$ in equation (19). Note also that to account for the traction that is applied to the surfaces of the crack by the fluid pressure (not included in the formulation presented), the solution derived in this section must be added to that found in Section 3 for a dry crack using in equations (18) and (19) $\mathrm{K}_{\mathrm{I}}=\left(\mathrm{u}_{\mathrm{o}}-\mathrm{u}_{\mathrm{ff}}\right) \sqrt{\pi \mathrm{a}}, \mathrm{K}_{\mathrm{II}}=0$.

As it was done in the previous two cases, namely dry and undrained cases, the analytical solution obtained is checked against the results obtained using the Finite Element code ABAQUS. The same cases are compared: a horizontal crack, $\beta=0$, Figures 8(a), 8(c) and 8(e); and a crack with $\beta=45^{\circ}$, Figures $8(\mathrm{~b}), 8(\mathrm{~d})$ and 8(f). In both cases a pore pressure of $1 \mathrm{MPa}$, and thus a total traction of $1 \mathrm{MPa}$, is applied at the surfaces of the crack that has a 
length $\mathrm{a}=1 \mathrm{~m}$, and no pore pressures and no tractions far from the crack. The rock properties are as follows: $\mathrm{E}_{\mathrm{x}}=7800 \mathrm{MPa}, \mathrm{E}_{\mathrm{y}}=2400 \mathrm{MPa}, v_{\mathrm{yx}}=0.07, v_{\mathrm{xz}}=0.22, v_{\mathrm{yz}}=0.02$,

$\mathrm{G}_{\mathrm{xy}}=830 \mathrm{MPa}, \alpha_{\mathrm{x}}=\alpha_{\mathrm{y}}=1$ and $\mathrm{M}>>1$. As we can see in Figure 8, the two results are comparable.

\section{Discussion}

It is interesting to investigate the stress field in a pressurized crack in an elastic transversely anisotropic rock. This is the situation that may occur inside a rock mass after a crack has been created due to hydraulic fracturing. This problem is idealized by considering an isolated crack in an infinite medium subjected only to internal pressure. The results are thus not intended to duplicate actual conditions, but rather to provide insight into the stress field of a pressurized crack under different loading conditions. The problem analyzed is similar to that considered for the verification of the analytical solutions. That is, a two-dimensional crack in an infinite elastic transversely anisotropic rock with the following properties: crack length $\mathrm{a}=1 \mathrm{~m}, \mathrm{E}_{\mathrm{x}}=7800 \mathrm{MPa}, \mathrm{E}_{\mathrm{y}}=2400 \mathrm{MPa}$, $v_{\mathrm{yx}}=0.07, v_{\mathrm{xz}}=0.22, v_{\mathrm{yz}}=0.02, \mathrm{G}_{\mathrm{xy}}=830 \mathrm{MPa}$ (the properties are those of the bedded sedimentary Waichecheng series, from Tonon and Amadei, 2002), and subjected to an internal pressure of $1 \mathrm{MPa}$; it is also assumed that $\alpha_{x}=\alpha_{y}=1$ and $M>>1$. Three cases are considered: dry rock mass, saturated rock mass and undrained loading (excess pore pressures are generated), and saturated rock mass with drained loading (no excess pore pressures are produced). Solutions are presented for the crack oriented parallel to the $\mathrm{X}$ axis $\left(\beta=0^{\circ}\right)$, at 45 degrees with the $x$-axis $\left(\beta=45^{\circ}\right)$ and parallel to the $y$-axis $\left(\beta=90^{\circ}\right)$. In addition, results are included for the case of isotropic medium. Note that there is no difficulty in using the formulation provided for isotropic conditions (we use $v_{\mathrm{yx}}=v_{\mathrm{xz}}=v_{\mathrm{yz}}=$ 0.2; note that the isotropic results are independent of the Young's modulus).

Figures 9 and 10 contain contour plots of the effective tangential stresses at the tip of the crack. The effective tangential stresses are taken as the representative stresses since there is arguably evidence that they may be able to predict propagation in tension (e.g. Erdogan and Sih, 1963; Bobet, 2000). The figures show that for the dry and drained cases, a stress concentration in tension is induced at the tip of the crack and, due to the symmetry that 
exists when the crack is parallel to one of the axes of elastic symmetry, the direction of the stress singularity in tension is parallel to the crack. Thus, given the premise of the maximum effective tangential stress criterion, it is expected that the crack would propagate along its own plane. When the crack is oriented at an angle with the axes of elastic symmetry, initiation may occur off-plane (see Figure 10(a) and (e)). A comparison between the magnitudes of the effective tangential stresses indicates that, in all cases, the crack in the dry medium has larger tensile stresses, and thus it could be expected that initiation would occur at a smaller applied pressure than in the saturated rock under drained loading. The crack orientation has also an effect on the magnitude of the effective tangential stress. It increases from the isotropic case to the $\beta=0^{\circ}$ case, and then it decreases with increasing $\beta, \square \square \square \square \square \square \square \square$ for the material properties investigated. The decrease is more pronounced for the drained case. The reason for the reduction of effective tangential stresses from the dry case to the drained loading case is the presence of seepage forces inside the rock. With drainage, a seepage flow occurs inside the rock, which in turn creates seepage forces that tend to induce compression in the direction of flow (Lambe and Whitman, 1969).

The results obtained under the assumption of undrained loading are quite different than the other cases. Figures 9 and 10 consistently indicate the creation of compressive stresses around the tip of the crack, except for the case with $\beta=90^{\circ}$ where a small tension ahead of the tip occurs. This observation may have important implications for crack initiation, again with the assumption of initiation due to the maximum effective tangential stress criterion. Since the presence of a compressive field at the tip of a crack tends to favor propagation of the crack in shear (Bobet, 2000), it seems that crack pressurization under undrained loading may induce initiation in shear or delay fracture initiation in tension until sufficient fluid is permeated into the rock such that a partial transition between undrained loading (with large excess pore pressures) and partial drained loading has occurred and sufficient stresses are developed at the tip.

\section{Summary and Conclusions}


The paper presents new analytical solutions for the singular stress and displacement fields in the vicinity of a isolated crack in a poroelastic transversely anisotropic medium. Three scenarios are considered: dry, undrained loading and drained loading. For the dry scenario, the crack is placed in a medium where there are no pore pressures, while in the other two scenarios, the medium is assumed to be saturated. Undrained loading occurs when the rate of application of the load is higher than the capacity of the medium to dissipate excess pore pressures and, at the limit, i.e. undrained loading conditions, no dissipation of excess pore pressures occurs. Drained loading is achieved when the rate of loading is relatively slow such that no excess pore pressures occur. In low permeability rock, e.g. shales, undrained conditions may occur at the very early stages of loading, while drained conditions are established some time after the application of the loading.

The solutions obtained have been compared with the results from the Finite Element code ABAQUS (2013). A number of cases have been investigated with a crack at different orientations in an elastic transversely anisotropic rock with the properties corresponding to those of the bedded sedimentary Waichecheng series (Tonon and Amadei, 2002); that is, $\mathrm{E}_{\mathrm{x}}=7800 \mathrm{MPa}, \mathrm{E}_{\mathrm{y}}=2400 \mathrm{MPa}, v_{\mathrm{yx}}=0.07, v_{\mathrm{xz}}=0.22, v_{\mathrm{yz}}=0.02, \mathrm{G}_{\mathrm{xy}}=830 \mathrm{MPa}$ and also assuming $\alpha_{\mathrm{x}}=\alpha_{\mathrm{y}}=1$ and $\mathrm{M}>>1$. All comparisons are satisfactory, which provide credibility to the formulation obtained.

A limited parametric analysis has been done using the closed-form expressions obtained. The analyses are intended to provide insight into the stresses produced at the tip of a crack under pressure. The three scenarios, namely dry, undrained and drained, are considered either in an isotropic medium or in a transversely anisotropic medium using the properties of the Waichecheng rock. The results, albeit limited in scope, point towards a decrease of stresses at the tip for drained conditions, compared to dry conditions. In all scenarios, undrained loading is associated with a large reduction of stresses, which in the majority of the cases are compressive due to the generation of large excess pore pressures at the tip. This suggests that some drainage may need to occur from the inside of the crack to the rock before propagation can occur. When the crack is oriented along the direction of one of the principal axes of elastic symmetry, the stresses are symmetric with respect to the plane of the crack. The symmetry is lost when the crack is oriented at an 
angle with the principal axes of anisotropy, which suggests that initiation may not occur along the plane of the crack.

\section{Acknowledgements}

This work was sponsored by the National Science Foundation, Geomechanics and Geotechnical Systems Program, under grant CMMI-1162082 to Purdue University and by the National Natural Science Foundation of China (51208296), the Fundamental Research Funds for the Central Universities (2013KJ095), Shanghai Educational Development Foundation (13CG17) and Kwang-Hua Education Fund of Tongji University. The authors are grateful to the sponsors for their support. 


\section{References}

ABAQUS (2013). User's Manual, Version 6.13-4. Dassault Systemes Simulia Corp., Providence, RI, USA.

Aköz, A.Y. and Tauchert, T.R. (1972). Thermal Stresses in an Orthotropic Elastic Semispace. Journal of Applied Mechanics, March Issue, pp. 87-90.

Atkinson, C. and Clemens, D.L. (1977). On Some Crack Problems in Anisotropic Thermoelasticity. International Journal of Solids and Structures, Vol. 31, pp. 855-864.

Bobet, A. and Einstein, H.H. (1998). Numerical Modeling of Fracture Coalescence in Rock Materials. International Journal of Fracture, Vol. 92, No. 3, pp. 221-252.

Bobet, A. (2000). The Initiation of Secondary Cracks in Compression. Engineering Fracture Mechanics, Vol. 66, No. 2, pp. 187-219.

Bobet, A., Fakhimi, A., Johnson, S., Morris, J., Tonon, F., and Yeung, M. (2009). Numerical Models in Discontinuous Media: A review of advances for rock mechanics applications. ASCE Journal of Geotechnical and Geoenvironmental Engineering, Vol. 135, No. 11, pp. 1547-1561.

Bobet, A. (2001). A Hybridized Displacement Discontinuity Method for Mixed Mode III-III Loading. International Journal of Rock Mechanics and Mining Sciences. Vol. 38, pp. 1121-1134.

Bobet, A. (2011). Lined Circular Tunnels in Transversely Anisotropic Rock at Depth. Rock Mechanics and Rock Engineering, Vol. 44, pp. 149-167.

Bobet, A., Garcia Marin, V. (2013). A Stress and Displacement Discontinuity Element Method for Elastic Transversely Anisotropic Rock. International Journal of Numerical and Analytical Methods in Geomechanics, Vol. 38, pp. 1898-1922.

Chen, C.-S., Pan, E. and Amadei, B. (1998). Fracture Mechanics Analysis of Cracked Discs of Anisotropic Rock Using the Boundary Element Method. International Journal of Rock Mechanics and Mining Sciences, Vol. 35, No. 2, pp. 195-218.

Cheng, A.H.-D. (1998). On Generalized Plan Strain Poroelasticity. International Journal of Rock Mechanics and Mining Sciences, Vol. 35, No. 2, pp. 183-193.

Clements, D.L. (1973). Thermal Stress in an Anisotropic Elastic Half-Space. SIAM Journal on Applied Mathematics, Vol. 24, No. 3, pp. 332-337.

Detournay, E. and Cheng, A.H.-D. (1993). Fundamentals of poroelasticity. In Comprehensive Rock Engineering: Principles, Practice and Projects. J.A. Hudson, editor. Pergamon Press, Oxford, UK, Vol. 2, pp. 113-171.

Erdogan, F. and Sih, G.C. (1963). On the Crack Extension in Plates under Loading and Transverse Shear. Journal of Basic Engineering, Vol. 85, pp. 519-527.

Griffith, A.A. (1920). The Phenomenon of Rupture and Flow in Solids. Philosophical Transactions of the Royal Society of London, Series A, Vol. 221, pp. 163-198.

Hedayat, A., Pyrak-Nolte, L.J. and Bobet, A. (2014). Seismic Precursors to the Shear Failure of Rock Discontinuities. Geophysical Research Letters, DOI: 10.1002/2014GL060848.

Ju, S.H., Chiu, C.Y. and Jhao, B.J. (2010).Determination of SIFs, crack-tip coordinates and crack angle of anisotropic materials. Fatigue and Fracture of Engineering Materials and Structures, Vol. 33, No. 1, pp. 43-53.

Kao, C., Schwanz, N., and Labuz, J. (2008) Progressive Failure of a Dam Abutment: A Fracture Mechanics Analysis. GeoCongress 2008: pp. 476-483. 
Labossiere, P.E.W. and Dunn M.L. (1998). Calculation of stress intensities at sharp notches in anisotropic media. Engineering Fracture Mechanics, Vol. 61, pp. 635-654.

Lambe, T.W. and Whitman, R.V. (1969). Soil Mechanics. John Wiley and Sons, Inc. USA.

Lekhnitskii, S.G. (1963). Theory of Elasticity of an Anisotropic Elastic Body. HoldenDay, Inc., San Francisco, CA.

Liebowitz, H. (1968). Fracture: An Advanced Treatise. Academic Press, New York.

McClintock, F.A. and Walsh, J.B. (1962). Friction of Griffith cracks in rock under pressure. Proceedings of the $4^{\text {th }}$ US National Congress on Applied Mechanics, Vol. II, ASCE New York, pp. 1015-1021.

Mutlu, O. and Bobet, A. (2006). Slip Propagation along Frictional Discontinuities. International Journal of Rock Mechanics and Mining Sciences, Vol. 43, pp. 860-876.

Sih, G.C. (1974). Strain-energy Density factor Applied to Mixed Mode Crack Problems. International Journal of Fracture, Vol. 10, No. 3, pp. 305-321.

Sih, G. C., Paris, P. C. and Irwin, G. R. (1965). On cracks in rectilinearly anisotropic bodies. International Journal of Fracture, Vol. 3, No. B7, pp. 189-203.

Su, R.K.L. and Sun, H.Y. (2003). Numerical solutions of two-dimensional anisotropic crack problems. International Journal of Solids and Structures, Vol. 40, pp. 46154635.

Ting, T.C.T. (1996). Anisotropic Elasticity: Theory and Applications. Oxford University Press, New York, NY.

Tinga, T. (2006). Stress intensity factors and crack propagation in a single crystal nickelbased superalloy. Engineering Fracture Mechanics, Vol. 73, pp. 1679-1692.

Tonon, F. and Amadei, B. (2002). Effect of Elastic Anisotropy on Tunnel Wall Displacements Behind a Tunnel Face. Rock Mechanics and Rock Engineering, Vol. 35, No. 3, pp. 141-160.

Wang, H.F. (2000). Theory of Linear Poroelasticity with Applications to Geomechanics and Hydrogeology. Princeton University Press, Princeton, NJ.

Whittaker, B.N., R.N. Singh, and G. Sun (1992). Rock Fracture Mechanics: Principles, Design and Applications. Elsevier Science Publishers, Amsterdam.

Wu, K.-C. and Chang, F.-T. (1993). Near-Tip Fields in a Notched Body With Dislocations and Body Forces. Transactions of the ASME, Vol. 60, pp. 936-941. 


\section{Figures}

Figure1

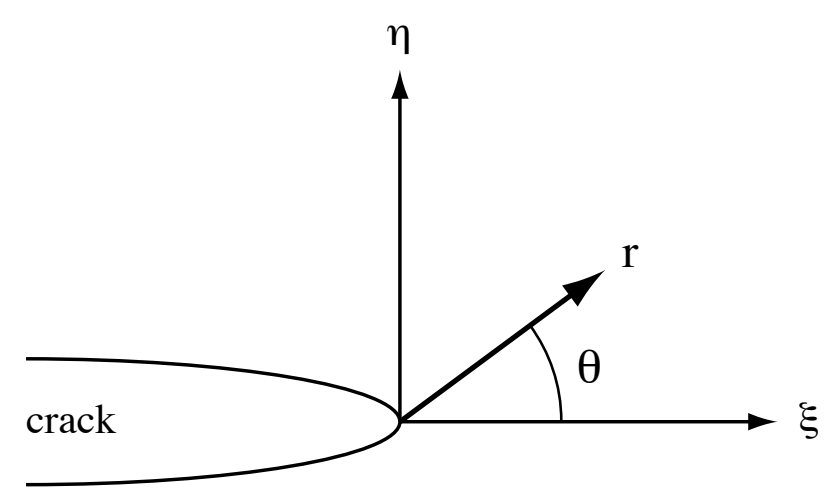

Figure 1. Cartesian and Polar Coordinates at the Tip of a Crack

Figure 1. Cartesian and Polar Coordinates at the Tip of a Crack

(1)

$\sqrt{2}$

$\sqrt{3}$

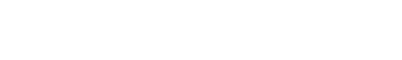

(n)




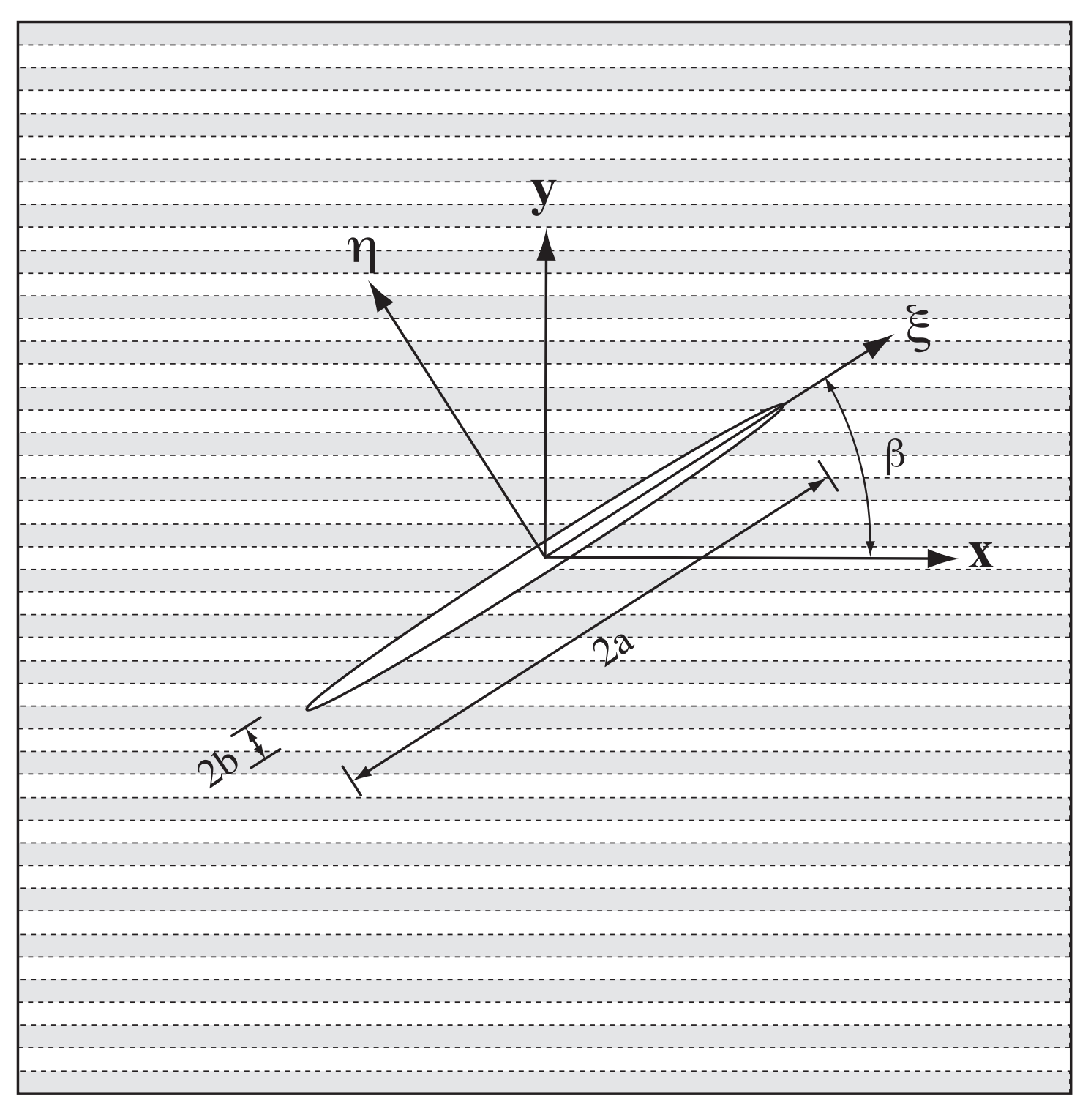

Figure 2. Crack in Transversely Anistropic Medium

Figure2

.

列

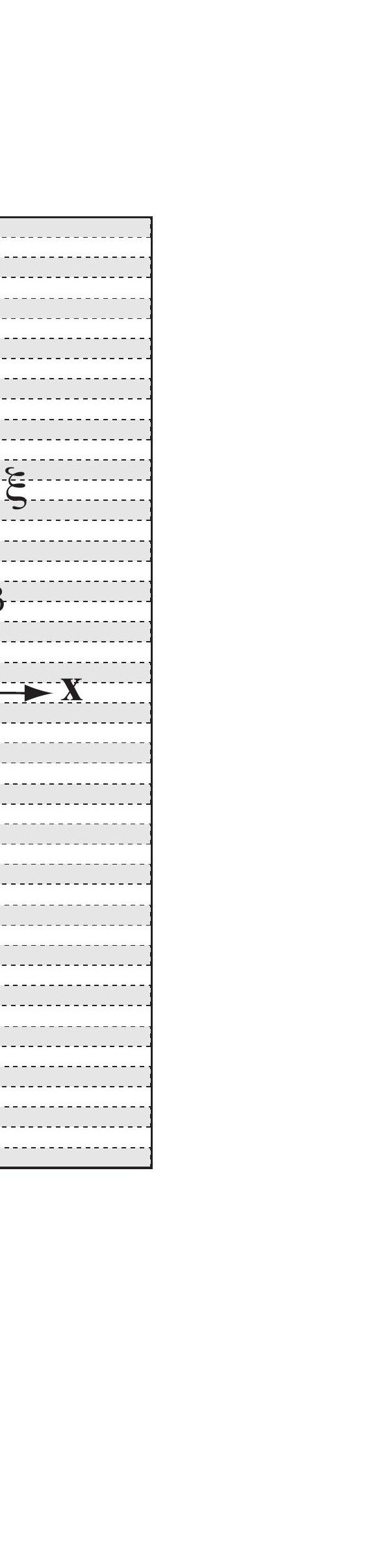

\title{
Figure2
}

\author{
The 2. Crack in Transversely Anistropic Medium
}

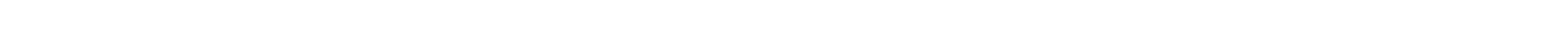

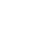
(1) 


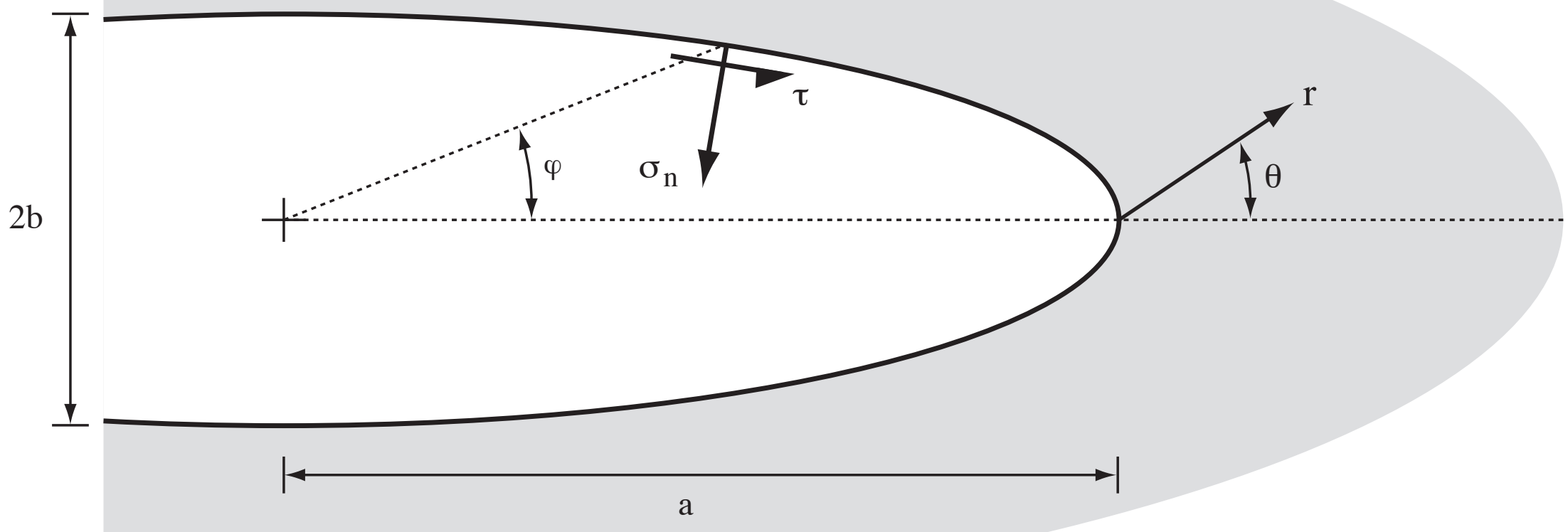

Figure 3. Stresses on the Crack Surface 


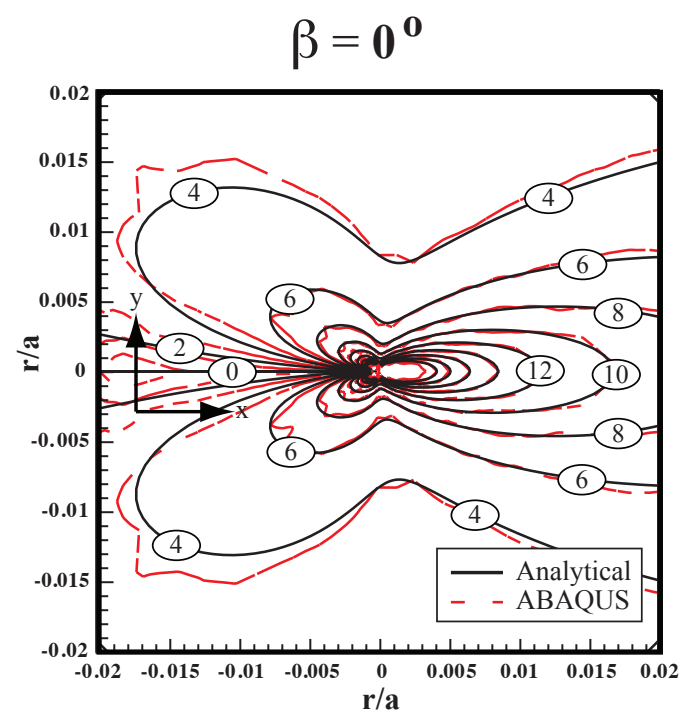

(a) $\sigma_{\mathrm{xx}}$

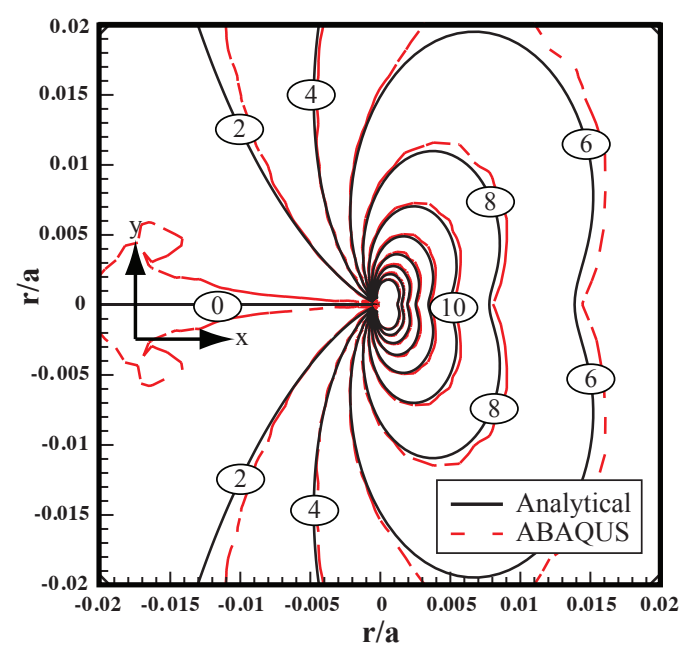

(c) $\sigma_{\text {yy }}$

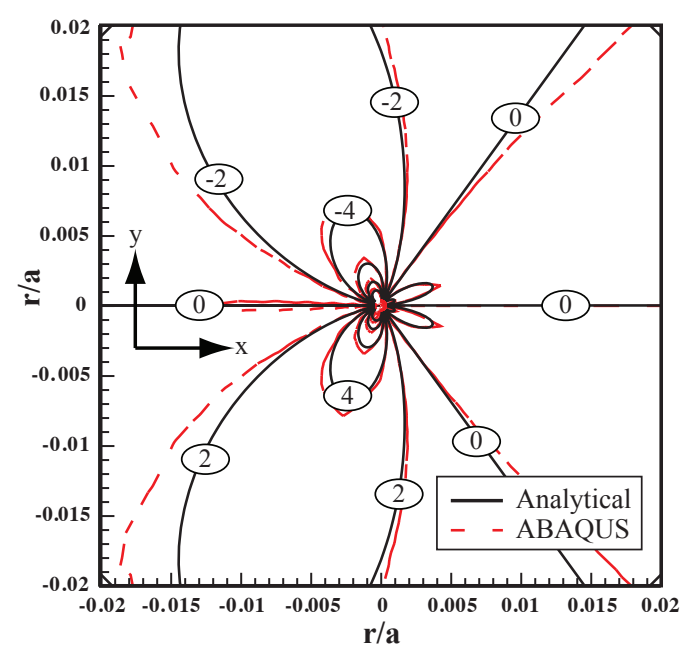

(e) $\sigma_{x y}$

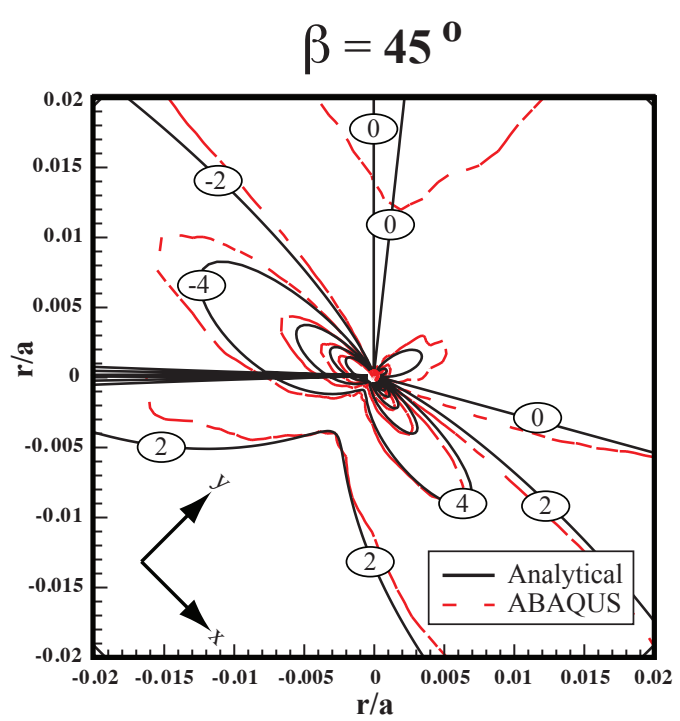

(b) $\sigma_{\mathrm{xx}}$

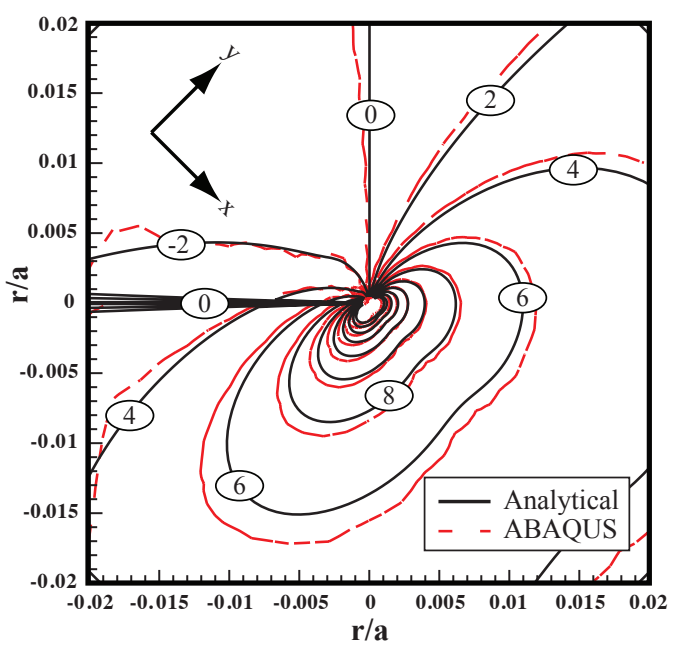

(d) $\sigma_{\text {yy }}$

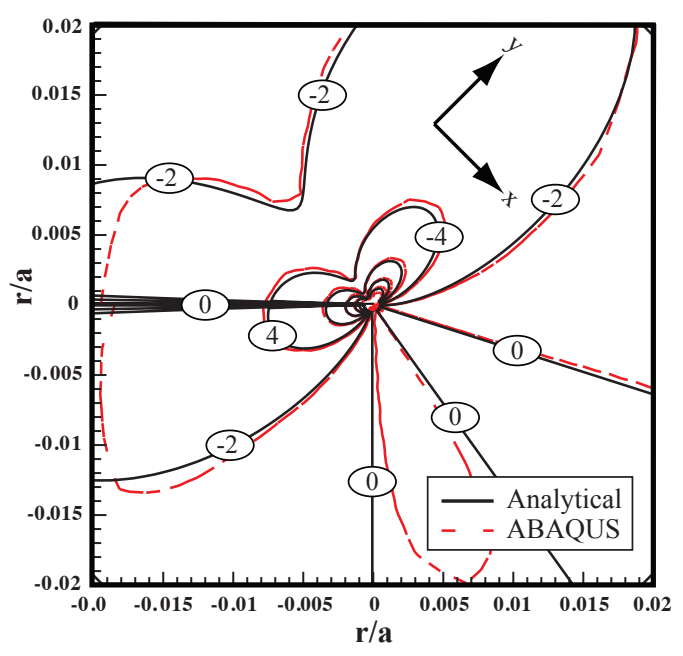

(f) $\sigma_{x y}$

Figure 4. Single Crack in an Infinite Medium. Dry Analysis. Comparison between Analytical Solution and ABAQUS; $a=1 \mathrm{~m}, \mathrm{E}_{\mathrm{x}}=7800 \mathrm{MPa}, \mathrm{E}_{\mathrm{y}}=2400 \mathrm{MPa}, \mathrm{G}_{\mathrm{xy}}=830 \mathrm{MPa}$, $v_{x z}=0.22, v_{y x}=0.07$, for Far-field Vertical Tensile Stress of 1 MPa. All values are in MPa. 


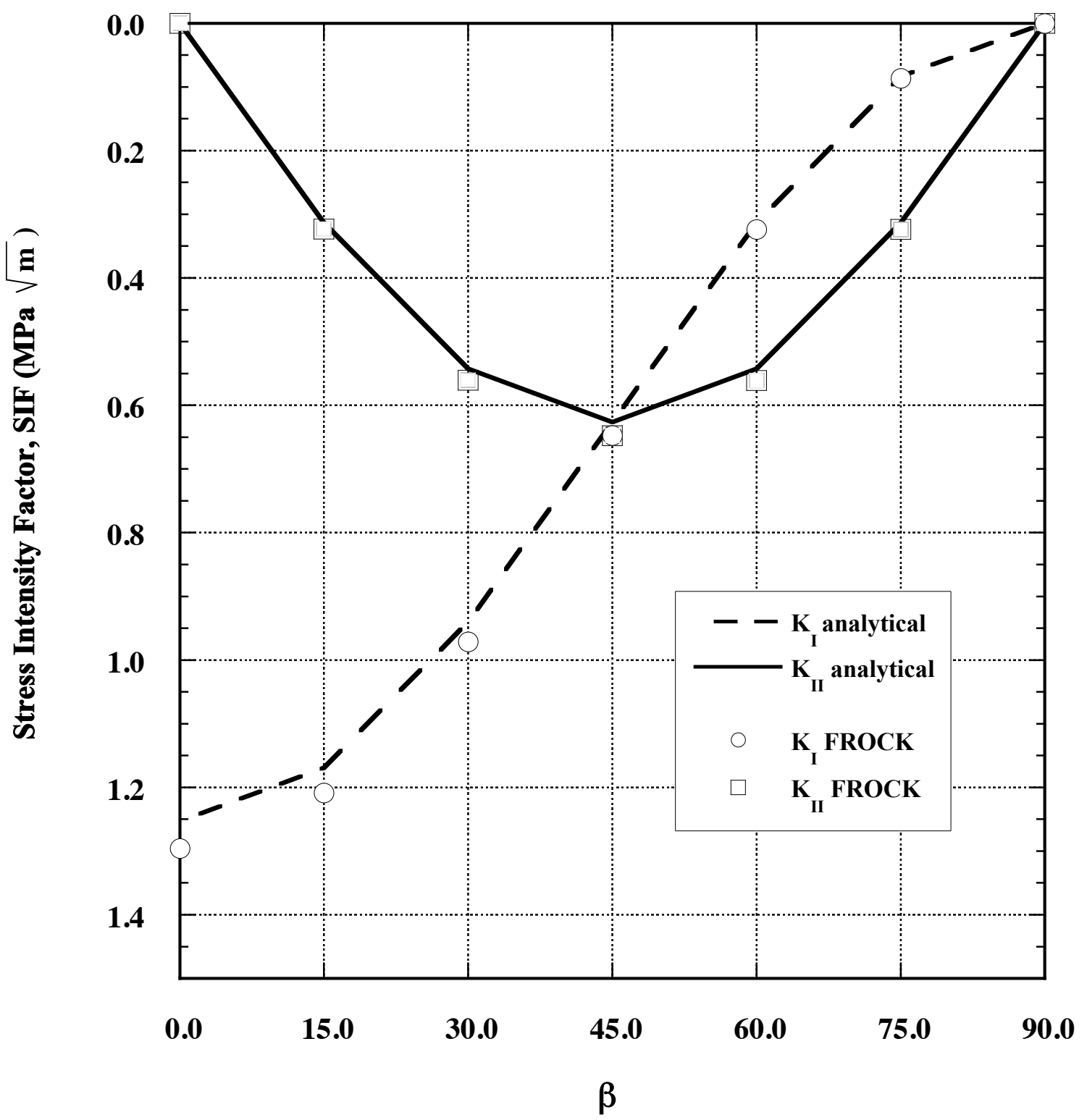

Figure 5. Comparison between Analytical and FROCK Stress Intensity Factors for a Single Open Crack in an Elastic Transversely Anisotropic Medium 


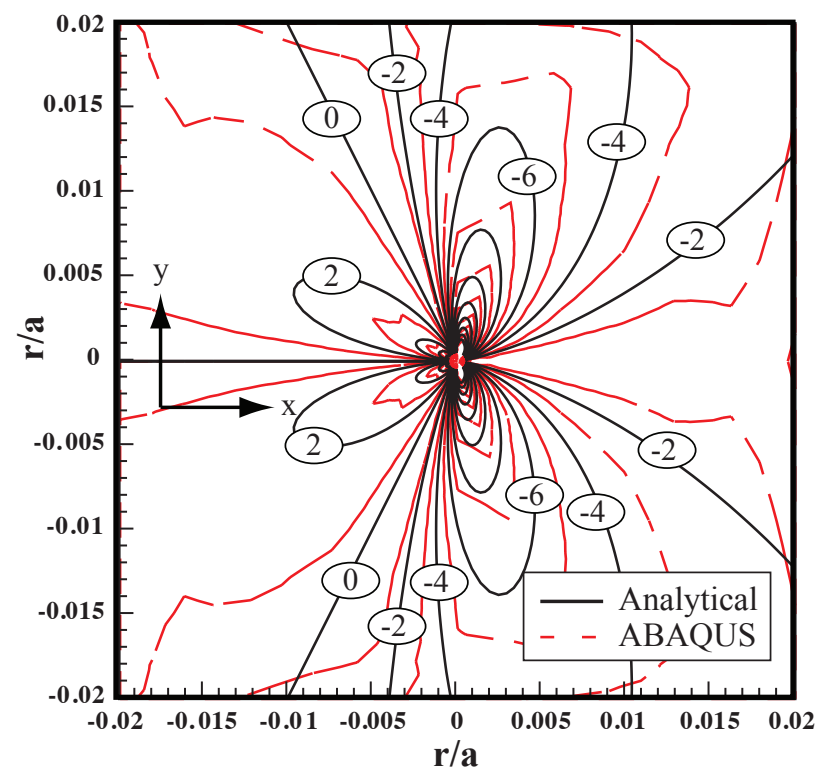

(a) $\sigma_{\mathrm{xx}}^{\prime}$

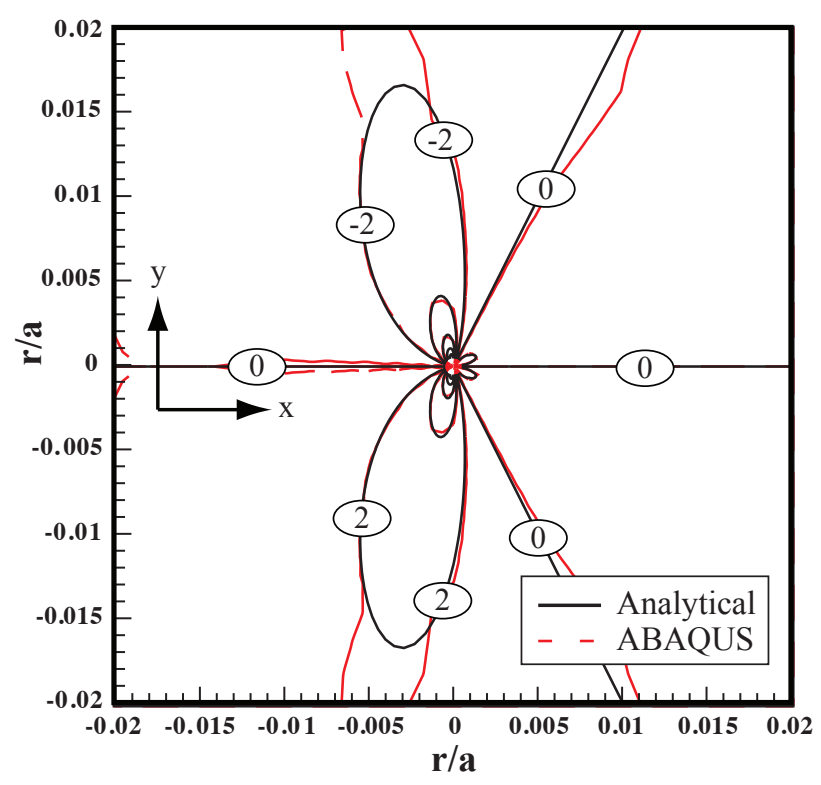

(c) $\sigma_{x y}$

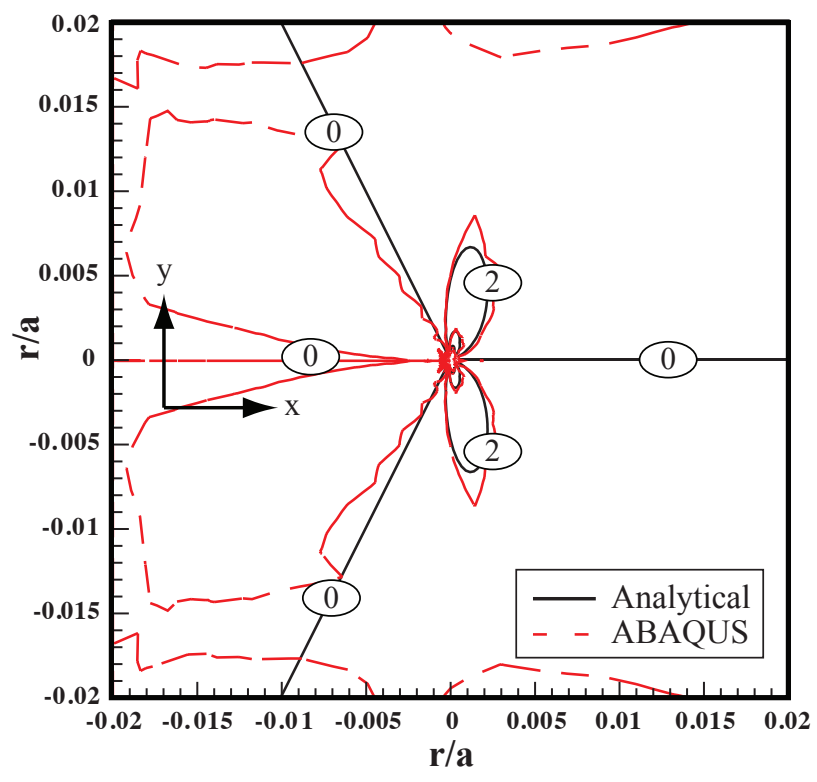

(b) $\sigma_{y y}^{\prime}$

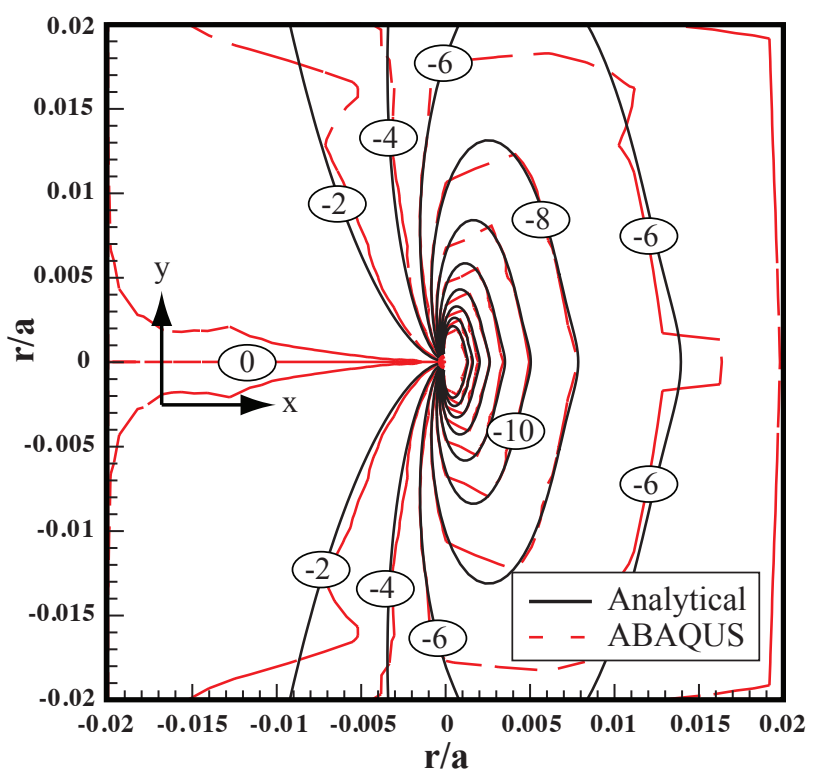

(d) $\Delta \mathbf{u}$

Figure 6. Single Crack in an Infinite Medium. Undrained Analysis. Comparison between Analytical Solution and ABAQUS; $a=1 \mathrm{~m}, \beta=0{ }^{\circ}, \mathrm{E}_{\mathrm{x}}=7800 \mathrm{MPa}, \mathrm{E}_{\mathrm{y}}=2400 \mathrm{MPa}, \mathrm{G}_{\mathrm{xy}}=830 \mathrm{MPa}, \nu_{\mathrm{xz}}=0.22, v_{\mathrm{yx}}=0.07$, and for a Far-field Vertical Tension Stress of 1 MPa. All values are in MPa. 


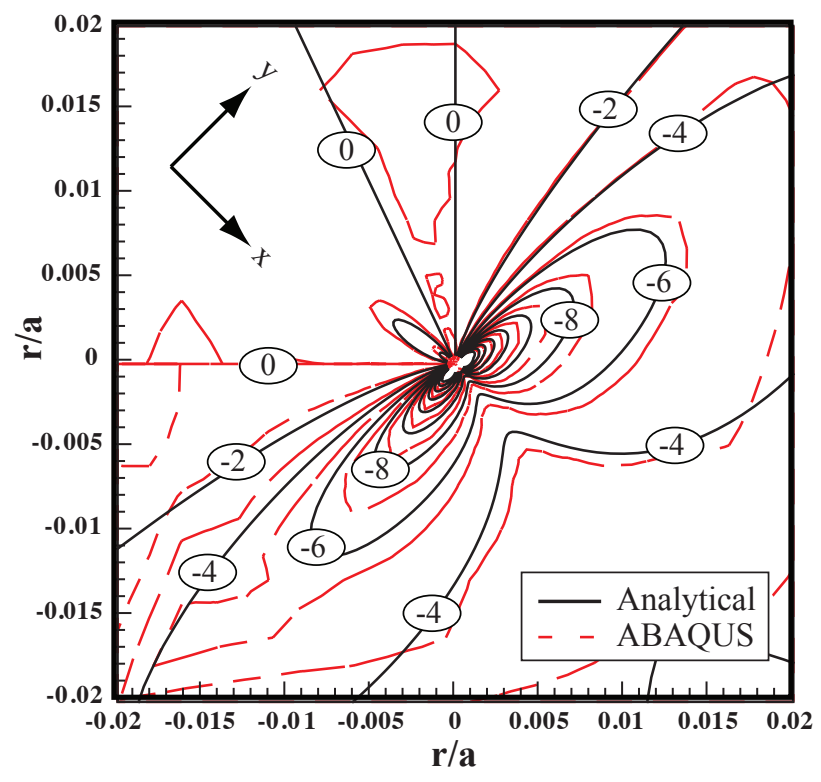

(a) $\sigma_{\mathrm{xx}}^{\prime}$

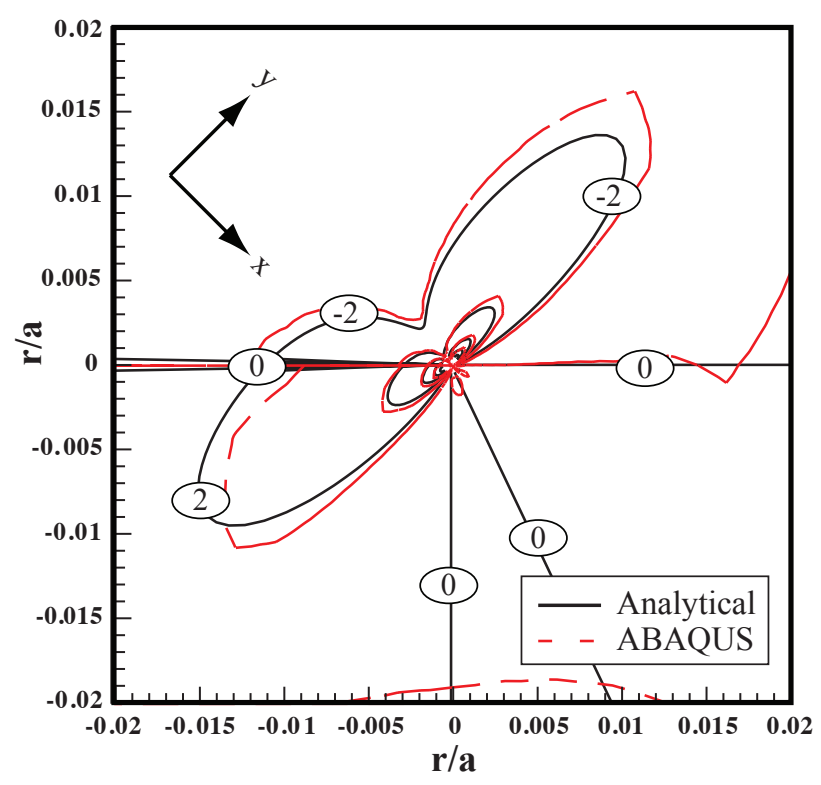

(c) $\sigma_{x y}$

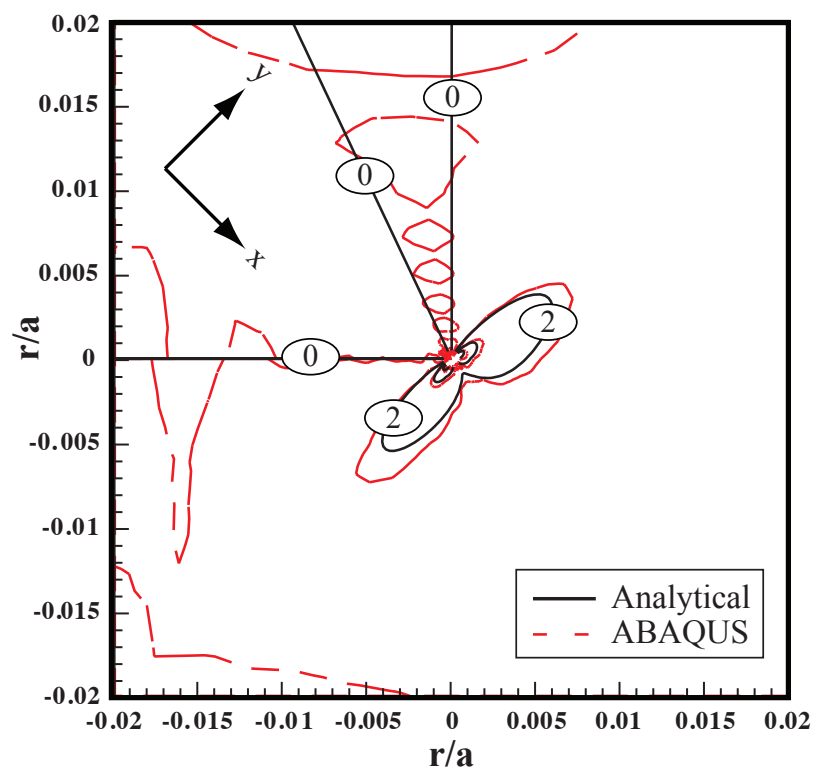

(b) $\sigma_{\mathrm{yy}}^{\prime}$

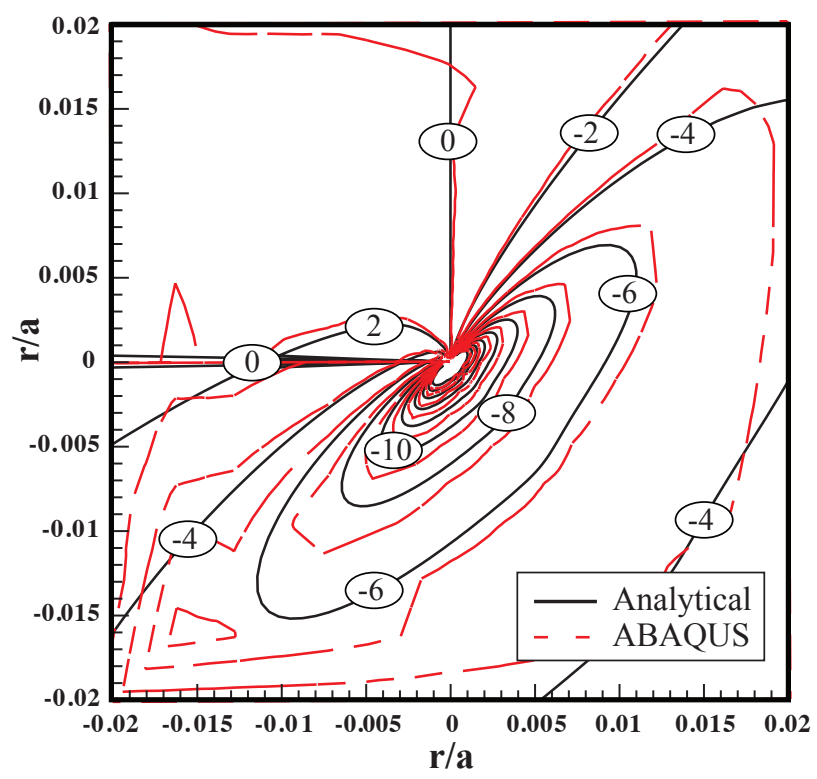

(d) $\Delta \mathbf{u}$

Figure 7. Single Crack in an Infinite Medium. Undrained Analysis. Comparison between Analytical Solution and ABAQUS; $a=1 \mathrm{~m}, \beta=45^{\circ}, \mathrm{E}_{\mathrm{x}}=7800 \mathrm{MPa}, \mathrm{E}_{\mathrm{y}}=2400 \mathrm{MPa}, \mathrm{G}_{\mathrm{xy}}=830 \mathrm{MPa}, \mathrm{v}_{\mathrm{xz}}=0.22, v_{\mathrm{yx}}=0.07$, and for a Far-field Vertical Tension Stress of 1 MPa. All values are in MPa. 


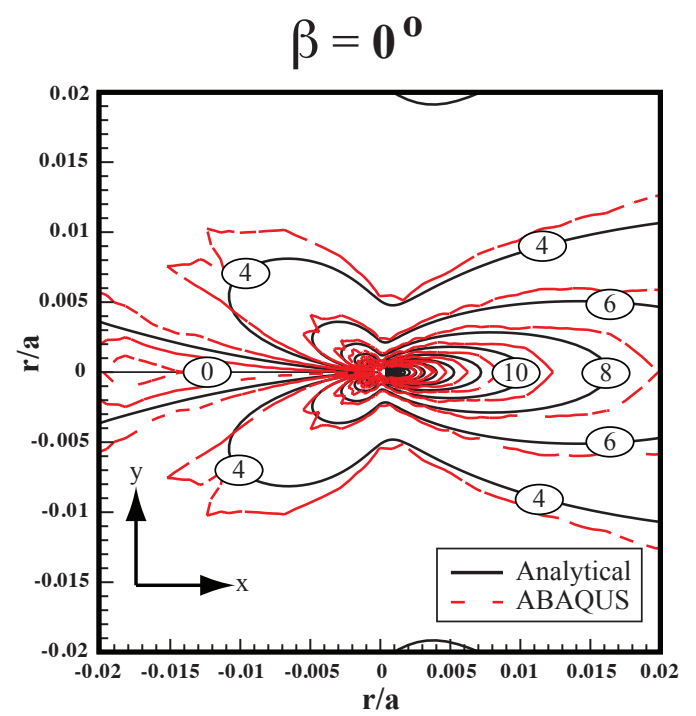

(a) $\sigma_{x x}$

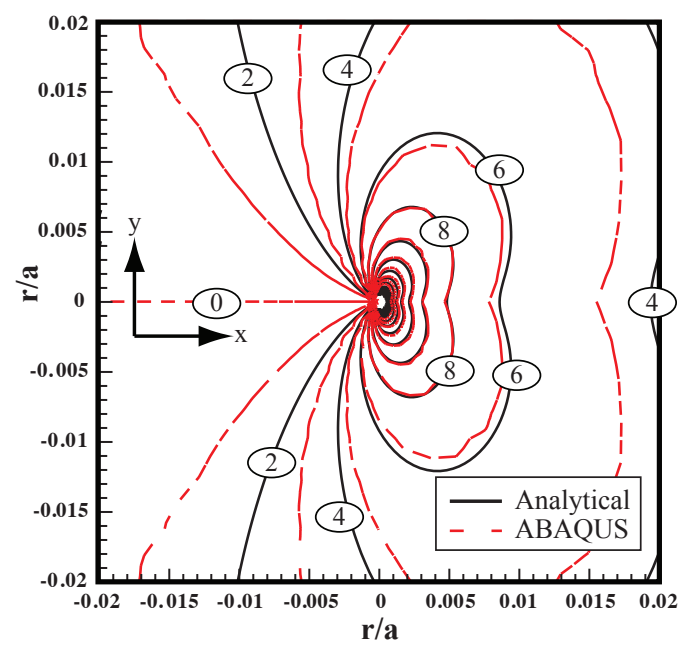

(c) $\sigma_{y y}$

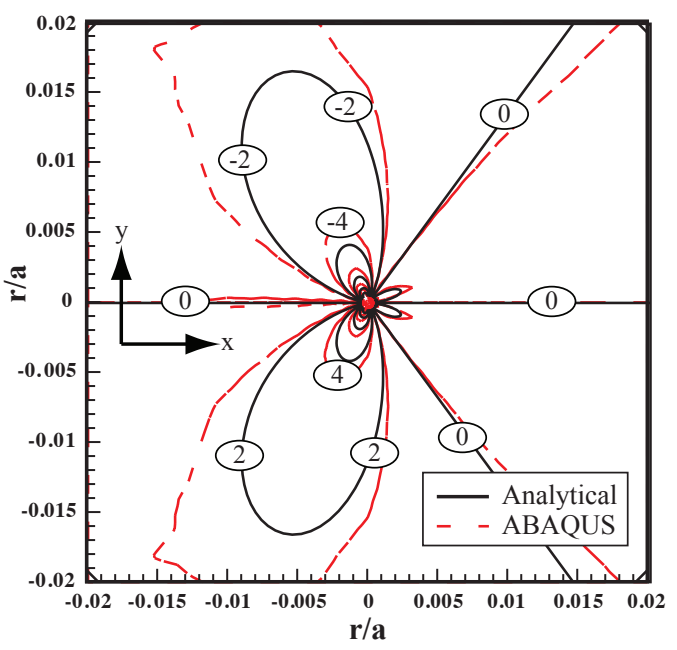

(e) $\sigma_{x y}$

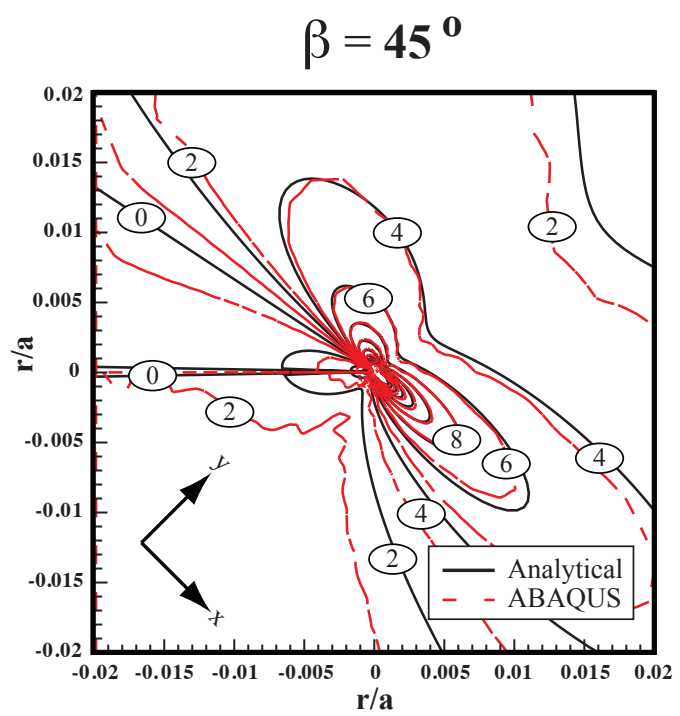

(b) $\sigma_{x x}$

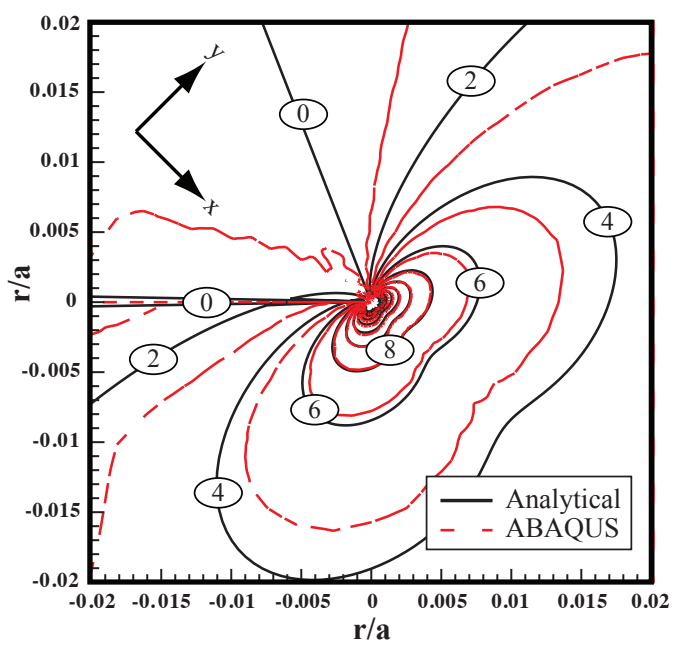

(d) $\sigma_{\text {yy }}$

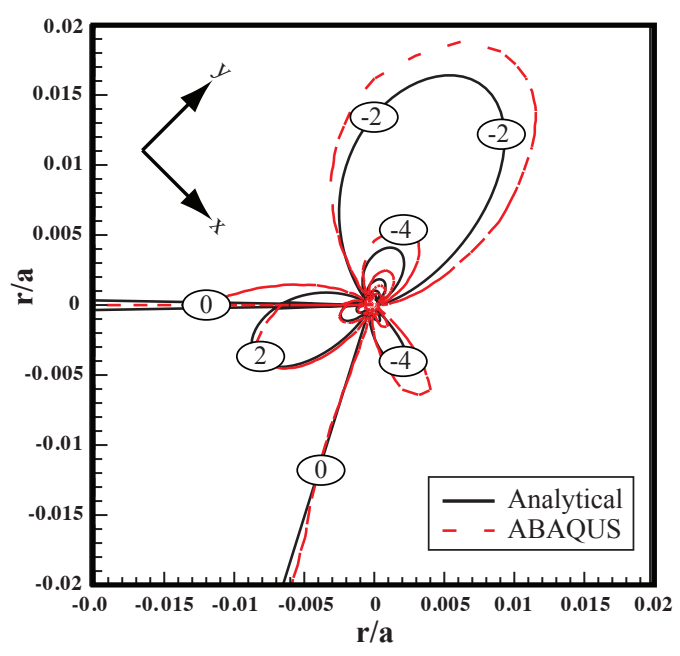

(f) $\sigma_{x y}$

Figure 8. Single Crack in an Infinite Medium. Drained Analysis. Comparison between Analytical Solution and ABAQUS; $a=1 \mathrm{~m}, \mathrm{E}_{\mathbf{x}}=7800 \mathrm{MPa}, \mathrm{E}_{\mathbf{y}}=2400 \mathrm{MPa}$, $G_{x y}=830 \mathrm{MPa}, v_{x z}=0.22, v_{y x}=0.07, u_{0}=1 \mathrm{MPa}, u_{f f}=0$. All values are in MPa. 
Isotropic

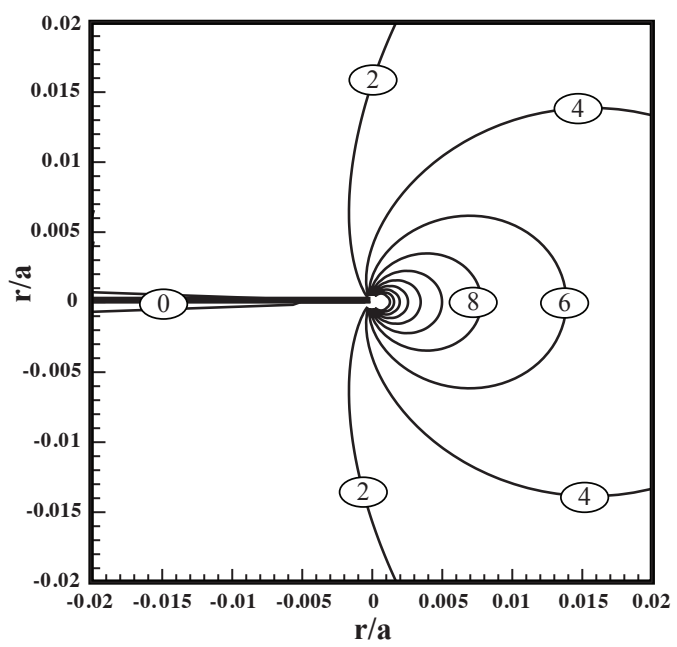

(a) Dry

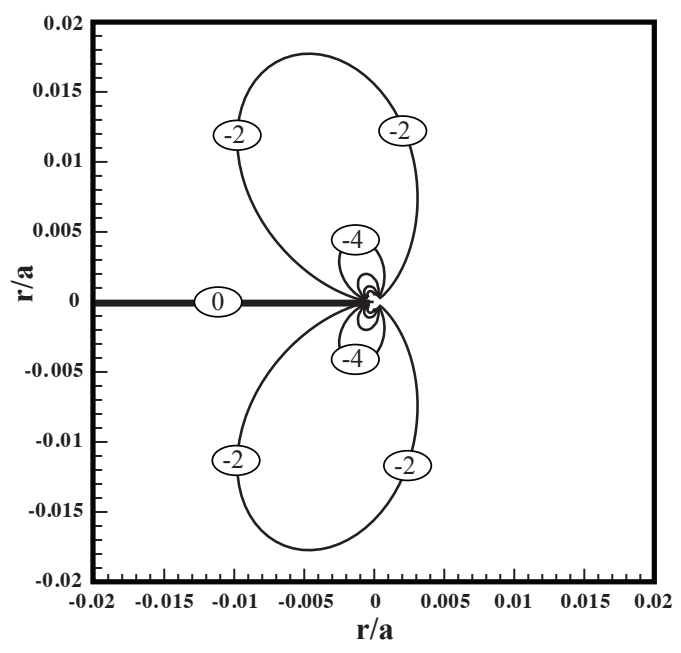

(c) Undrained

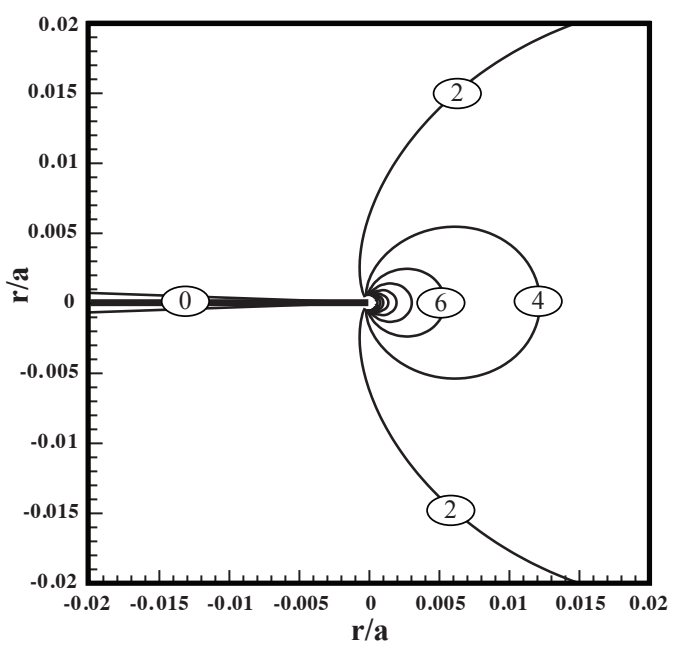

(e) Drained

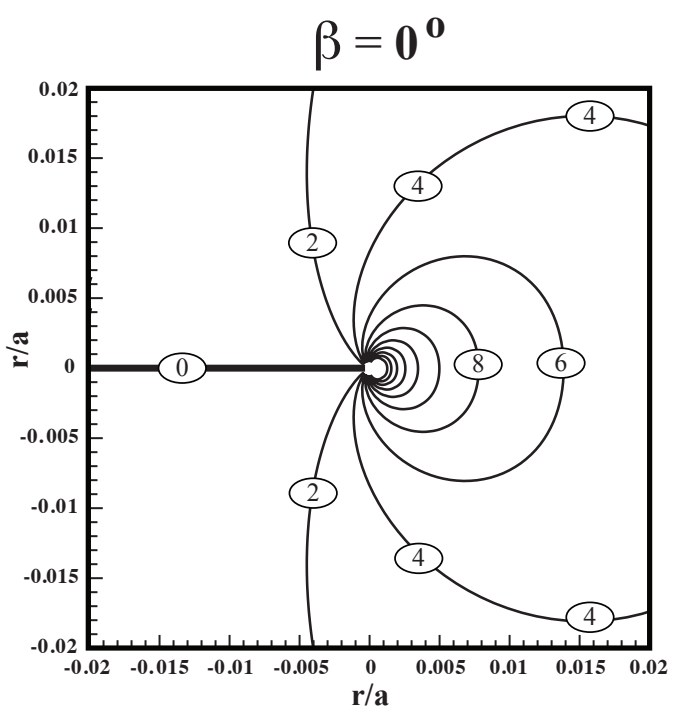

(b) Dry

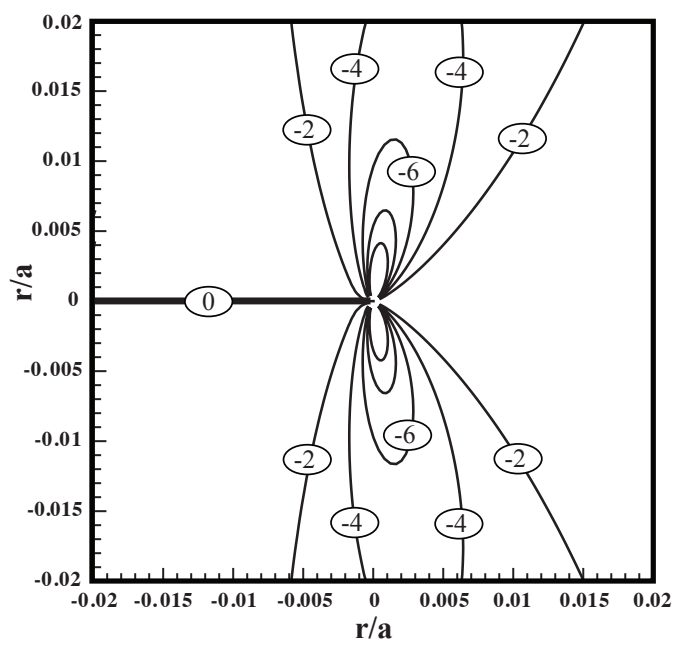

(d) Undrained

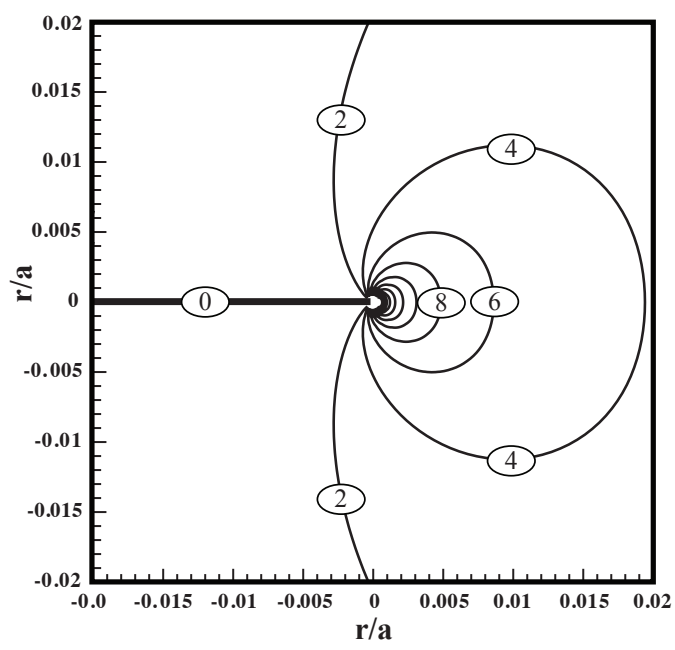

(f) Drained

Figure 9. Tangential Stresses (MPa) for Pressurized Single Crack in an Infinite Medium. Comparison between Dry, Undrained and Drained Cases, for: $a=1 \mathrm{~m}, \mathrm{E}_{\mathbf{x}}=7800 \mathrm{MPa}$, $E_{y}=2400 \mathrm{MPa}, G_{x y}=830 \mathrm{MPa}, v_{x z}=0.22, v_{y x}=0.07,(v=0.20$ drained $), u_{0}=1 \mathrm{MPa}, u_{f f}=0$. 


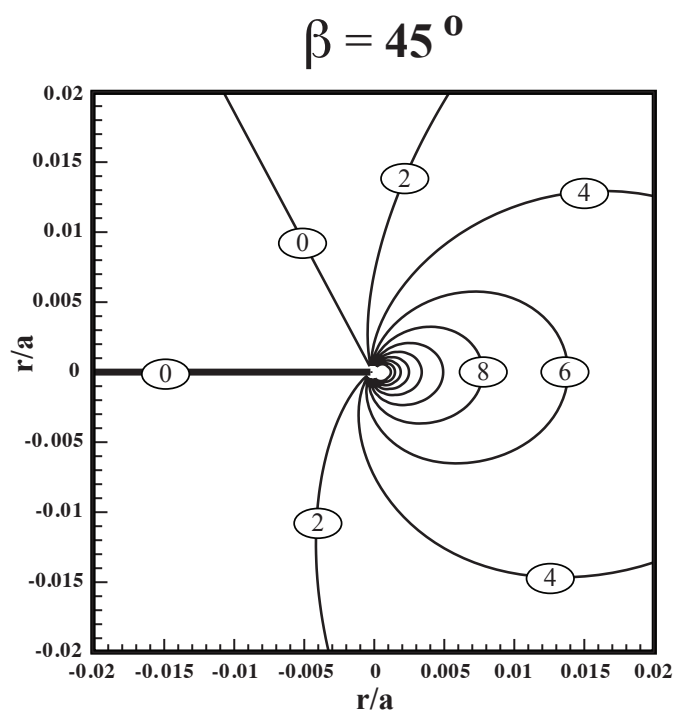

(a) Dry

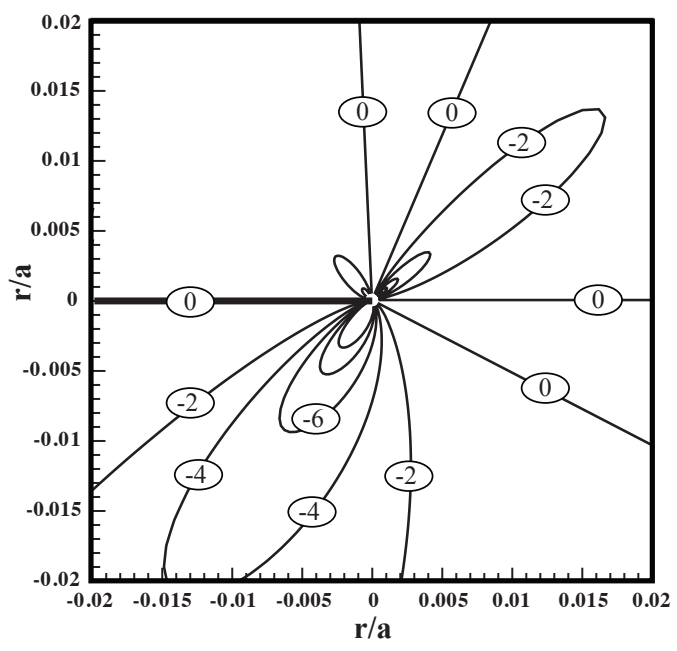

(c) Undrained

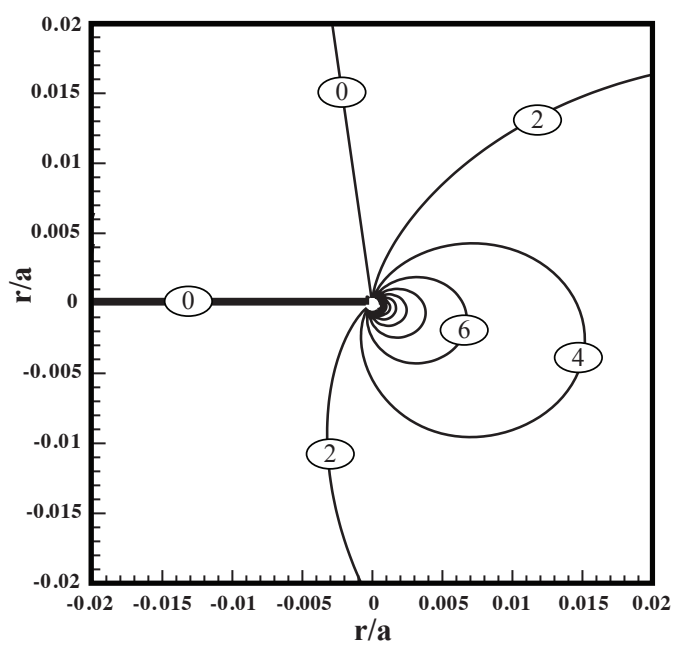

(e) Drained

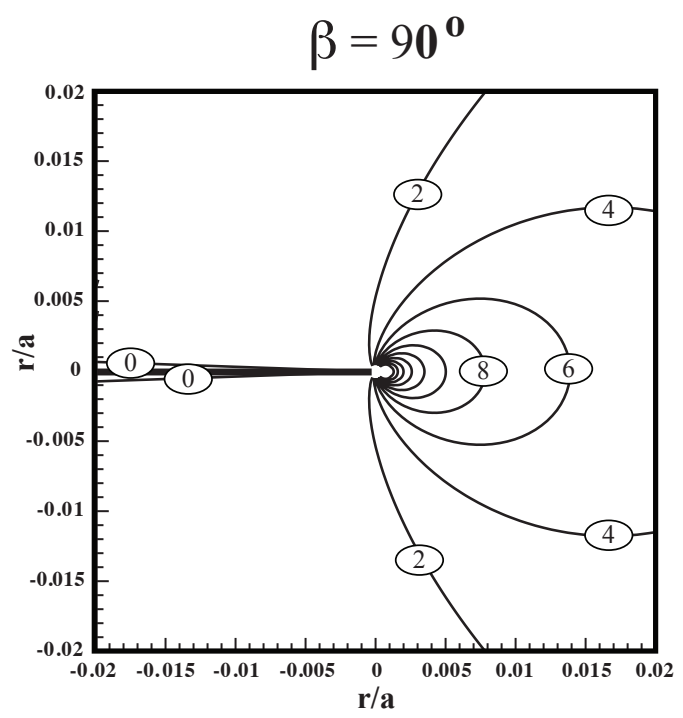

(b) Dry

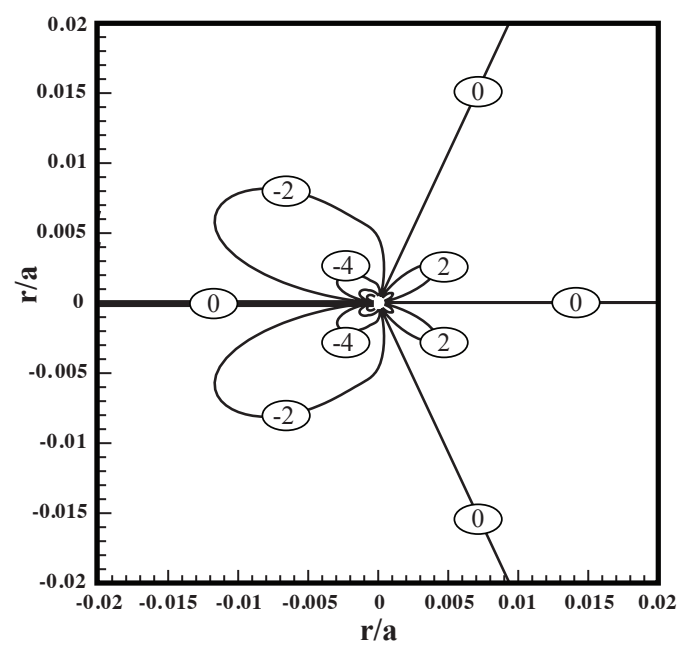

(d) Undrained

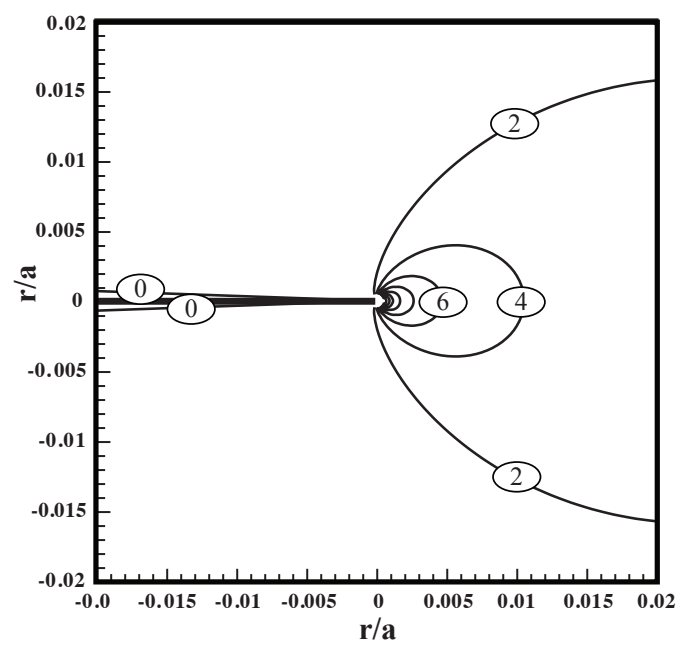

(f) Drained

Figure 10. Tangential Stresses (MPa) for Pressurized Single Crack in an Infinite Medium. Comparison between Dry, Undrained and Drained Cases, for: $a=1 \mathrm{~m}, \mathrm{E}_{\mathbf{x}}=7800 \mathrm{MPa}$, $E_{y}=2400 \mathrm{MPa}, G_{x y}=830 \mathrm{MPa}, v_{x z}=0.22, v_{y x}=0.07,(v=0.20$ drained $), u_{0}=1 \mathrm{MPa}, u_{f f}=0$. 